University of Tennessee Health Science Center

UTHSC Digital Commons

\title{
Use of a Multi-Axis Robotic Testing Platform to Investigate the Sagittal Mechanics of the Multi-Body Lumbar Spine
}

Jessica Rose Stubbs

University of Tennessee Health Science Center

Follow this and additional works at: https://dc.uthsc.edu/dissertations

Part of the Equipment and Supplies Commons, and the Investigative Techniques Commons

\section{Recommended Citation}

Stubbs, Jessica Rose, "Use of a Multi-Axis Robotic Testing Platform to Investigate the Sagittal Mechanics of the Multi-Body Lumbar Spine" (2011). Theses and Dissertations (ETD). Paper 256. http://dx.doi.org/ 10.21007/etd.cghs.2011.0302.

This Thesis is brought to you for free and open access by the College of Graduate Health Sciences at UTHSC Digital Commons. It has been accepted for inclusion in Theses and Dissertations (ETD) by an authorized administrator of UTHSC Digital Commons. For more information, please contact jwelch30@uthsc.edu. 


\title{
Use of a Multi-Axis Robotic Testing Platform to Investigate the Sagittal Mechanics of the Multi-Body Lumbar Spine
}

\begin{abstract}
A biomechanical study was performed to compare range of motion of the multi-body lumbar spine using three different protocols: pure moment, eccentric loading, and a new method called combined loading moment. The objectives of the study were to introduce a new protocol that overcomes the limitations of previous methods by applying more realistic loading conditions and to compare the range of motion of this new protocol to those of eccentric loading and pure moment protocols within the same specimen pool. The second objective of this study was to compare the data sets of these three protocols to both in vivo and in vitro data sets.

Because of the clinical issues with the lumbar region of the spine, it is important to understand the normal mechanics of the lumbar spine. In vivo methods of determining normal mechanics are limited, and in vitro methods have shown to be more realistic. Pure moment methods apply only a pure moment that is constant at every level of the spine. Pure moment with the addition of follower load increases the load carrying capacity of the in vitro spine. Eccentric loading produces physiological motion but the moment loads applied at each level are unknown. The previous methods of loading do not account for shear loads applied in vivo. A new method was developed called combined loading moment and includes axial, shear, and moment loading.

This study compared three methods using six harvested spines tested in flexion and extension. Eccentric loading was first applied using an existing protocol of the University of Tennessee Health Science Center (UTHSC) Joint Implant Biomechanics Laboratory. Pure moment and combined loading moment were both applied using new protocols adapted from pure moment for single motion segment units using the UTHSC Spine Robot. The intersegmental rotations were measured using a camera system with LEDs.

These rotations were used to create motion profiles to compare to in vivo studies and previous in vitro studies. Variations among in vivo and in vitro motion profiles could be due to a number of factors including subject groups and test methods.

Results from this study showed that follower load may not be necessary in order to apply in vivo loads to the in vitro spine. Axial load was determined to limit range of motion. Shear loads appeared to increase flexion range of motion, but appeared to only increase extension range of motion until the facets joints prevented further motion. The pure moment protocol used with the Spine Robot overcame the limitations of standard fixed pulley methods of pure moment. Future work with the new protocol should attempt to increase the magnitude of the vertical load and further explore the effects of shear load. Additionally, future work should include lateral, axial, and coupled rotations.
\end{abstract}

\section{Document Type}

Thesis

Degree Name

Master of Science (MS)

Program

Biomedical Engineering and Imaging

Research Advisor

Denis J. DiAngelo, Ph.D. 


\section{Keywords}

In vitro testing, multi-body lumbar spine, range of motion, intersegmental rotation

\section{Subject Categories}

Analytical, Diagnostic and Therapeutic Techniques and Equipment | Equipment and Supplies | Investigative Techniques | Medicine and Health Sciences 
USE OF A MULTI-AXIS ROBOTIC TESTING PLATFORM TO INVESTIGATE THE SAGITTAL MECHANICS OF THE MULTI-BODY LUMBAR SPINE

\author{
A Thesis \\ Presented for \\ The Graduate Studies Council \\ The University of Tennessee \\ Health Science Center
}

\begin{abstract}
In Partial Fulfillment
Of the Requirements for the Degree

Master of Science

In the Joint Graduate Program in Biomedical Engineering and Imaging

From The University of Tennessee

and

The University of Memphis
\end{abstract}

By

Jessica Rose Stubbs

May 2011 
Copyright (C) 2011 by Jessica Rose Stubbs.

All rights reserved. 


\section{DEDICATION}

For my grandparents.

To my grandpa John F. Stubbs for paving an engineering path and my grandmother Betty Stubbs for her loving support of his path.

To my papa Dr. Robert E. Mangum for his inspiration in higher education and my gran Jeri Mangum for all the much needed rice krispie treats. 


\section{ACKNOWLDEGEMENTS}

I would like to thank everyone that helped make my graduate experience enjoyable and productive. First, I would like to thank my advisor, Dr. Denis DiAngelo, for his guidance and patience. I would like to thank my committee members, Dr. Brian Kelly and Dr. Gladius Lewis for their input on this research. For the lumbar images from his model, I must also thank Dr. Yuan Li. Kyle Fraysur, Braham Dhillon, Karen Sedacki, and Dan Wido also deserve special thanks for all their help with all the projects I worked on while in the lab. I want to thank all the members of the Joint Implant and Biomechanics Lab.

I must also thank my family for all their encouragement, especially my father and stepmom. Finally, I must thank my generous mother for her incredible support

throughout my academic career. Words cannot express my appreciation for all her help and encouragement. 


\begin{abstract}
A biomechanical study was performed to compare range of motion of the multibody lumbar spine using three different protocols: pure moment, eccentric loading, and a new method called combined loading moment. The objectives of the study were to introduce a new protocol that overcomes the limitations of previous methods by applying more realistic loading conditions and to compare the range of motion of this new protocol to those of eccentric loading and pure moment protocols within the same specimen pool. The second objective of this study was to compare the data sets of these three protocols to both in vivo and in vitro data sets.
\end{abstract}

Because of the clinical issues with the lumbar region of the spine, it is important to understand the normal mechanics of the lumbar spine. In vivo methods of determining normal mechanics are limited, and in vitro methods have shown to be more realistic. Pure moment methods apply only a pure moment that is constant at every level of the spine. Pure moment with the addition of follower load increases the load carrying capacity of the in vitro spine. Eccentric loading produces physiological motion but the moment loads applied at each level are unknown. The previous methods of loading do not account for shear loads applied in vivo. A new method was developed called combined loading moment and includes axial, shear, and moment loading.

This study compared three methods using six harvested spines tested in flexion and extension. Eccentric loading was first applied using an existing protocol of the University of Tennessee Health Science Center (UTHSC) Joint Implant Biomechanics Laboratory. Pure moment and combined loading moment were both applied using new protocols adapted from pure moment for single motion segment units using the UTHSC Spine Robot. The intersegmental rotations were measured using a camera system with LEDs. These rotations were used to create motion profiles to compare to in vivo studies and previous in vitro studies. Variations among in vivo and in vitro motion profiles could be due to a number of factors including subject groups and test methods.

Results from this study showed that follower load may not be necessary in order to apply in vivo loads to the in vitro spine. Axial load was determined to limit range of motion. Shear loads appeared to increase flexion range of motion, but appeared to only increase extension range of motion until the facets joints prevented further motion. The pure moment protocol used with the Spine Robot overcame the limitations of standard fixed pulley methods of pure moment. Future work with the new protocol should attempt to increase the magnitude of the vertical load and further explore the effects of shear load. Additionally, future work should include lateral, axial, and coupled rotations. 


\section{TABLE OF CONTENTS}

CHAPTER 1. INTRODUCTION ........................................................................................1

CHAPTER 2. BACKGROUND OF THE LUMBAR SPINE ......................................3

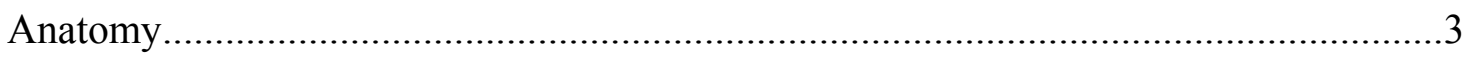

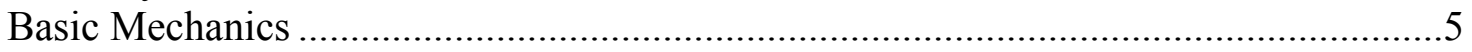

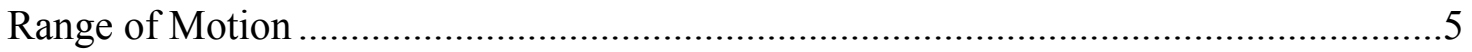

CHAPTER 3. IN VITRO TESTING METHODOLOGIES OF THE MULTI-

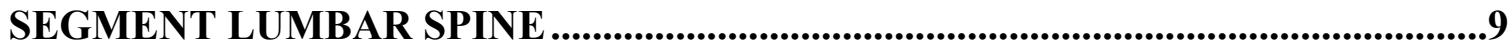

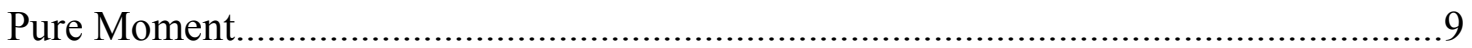

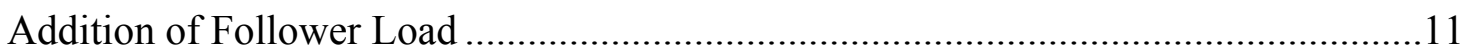

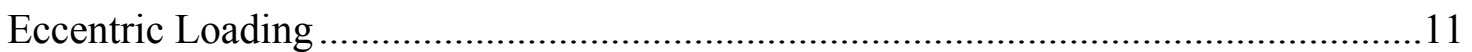

Combined Loading Moment: A New Method ............................................................14

\section{CHAPTER 4. AN IN VITRO INVESTIGATION OF MULTI-SEGMENT}

LUMBAR RANGE OF MOTION USING THREE TESTING PROTOCOLS ........17

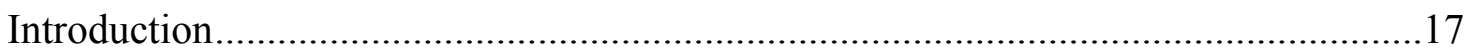

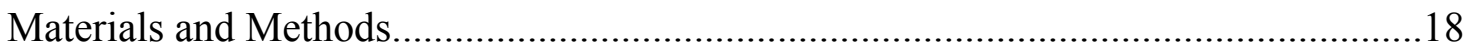

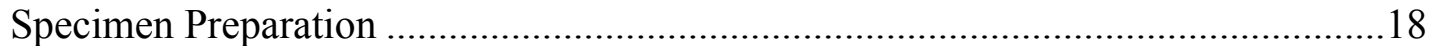

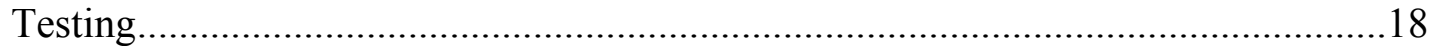

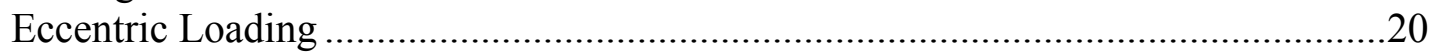

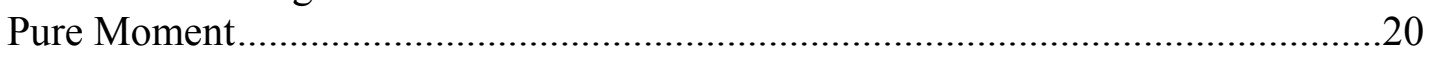

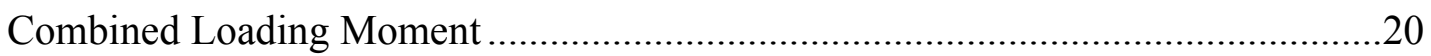

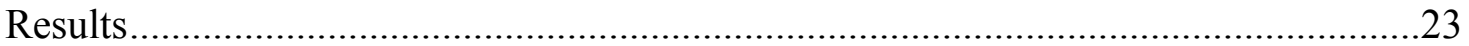

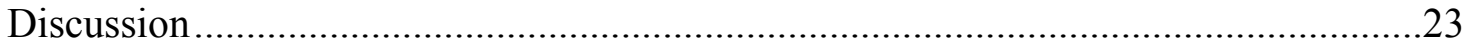

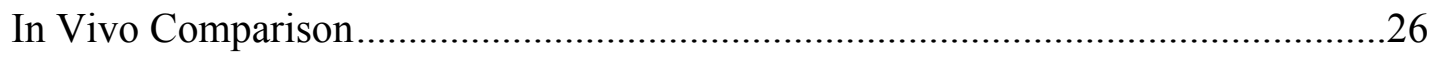

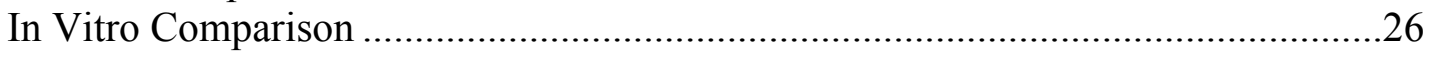

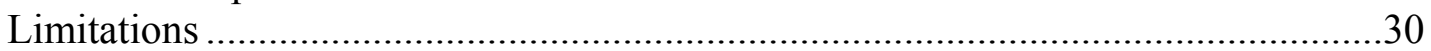

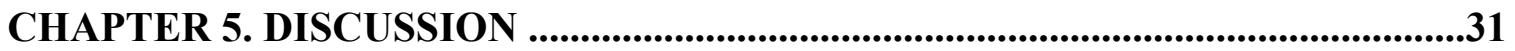

CHAPTER 6. LIMITATIONS, CONCLUSIONS, AND FUTURE WORK..............35

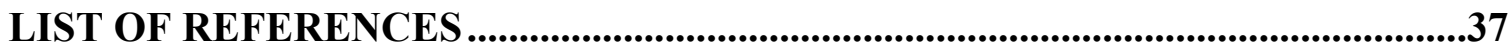

APPENDIX A. RADIOGRAPHS .....................................................................................40

APPENDIX B. FLEXIBILITY CURVES ..........................................................41

VITA 


\section{LIST OF TABLES}

Table 4-1. Average intersegmental and global rotations in degrees and standard deviations.

Table 5-1. Comparison of various methods of measuring intersegmental rotations in degrees. 


\section{LIST OF FIGURES}

Figure 2-1. The vertebra of the lumbar spine ..........................................................

Figure 2-2. The force of body weight on the lumbar spine. .............................................6

Figure 2-3. Degrees of freedom in two dimensions and three dimensions. ......................7

Figure 3-1. Diagram of pure moment experimental setup using pulleys. .......................10

Figure 3-2. Depiction of compressive follower load. .......................................................12

Figure 3-3. Eccentric loading diagram of setup and loads. ...........................................13

Figure 3-4. Spine robot motion during PM. …………...............................................

Figure 3-5. Free body diagram of the new methodology CLM......................................16

Figure 4-1. Schematic drawing and summary of loads for each protocol.......................19

Figure 4-2. Test setup for EL protocol. ...............................................................21

Figure 4-3. Test setup for PM and CLM with force frame transformations for each

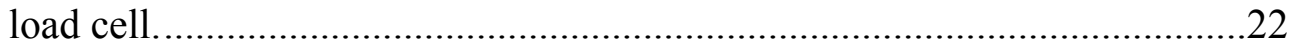

Figure 4-4. Comparisons of average global rotations and \%global motion profiles.......24

Figure 4-5. The relationship between shear force and rotation for one specimen during flexion and extension under Combined Loading Moment.................27

Figure 4-6. Comparison of the present study to previous in vivo studies. .....................28

Figure 4-7. Comparison of the present study to previous in vitro studies and the

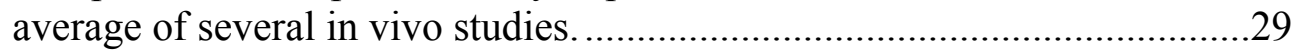

Figure A-1. Radiographs of specimens B, C, E, F, G, and $\mathrm{H}$ after rod placement...........40

Figure B-1. Flexibility curves for each specimen during flexion.....................................41

Figure B-2. Flexibility curves for each specimen during extension.................................42 


\section{LIST OF ABBREVIATIONS}

ALE

CLM

EL

FL

LED

LVDT

PM

ROM

RVDT
Adjacent Level Effects

Combined Loading Moment

Eccentric Loading

Follower Load

Light-Emitting Diode

Linear Variable Differential Transducer

Pure Moment

Range of Motion

Rotary Variable Differential Transducer 


\section{CHAPTER 1. INTRODUCTION}

The lumbar spine supports the largest amount of load on the spine and is thus believed to be the most injury prone [1]. Abnormal motion may be a key indicator of abnormal mechanics of the spine which is associated with back pain [2]. In order to determine these abnormal motions, knowledge of normal motion is necessary. Most in vivo methods of determining normal motion use only lateral images of subjects which limit measurement of motion to the sagittal plane [3-7]. The data sets reported from in vivo are not very consistent due to image quality, active versus passive bending, positioning of the subject, and other differences between testing methods. Also, the exact loads applied to the spine during in vivo measurements are not known. In vitro methods allow a more controlled environment for measuring range of motion and may actually be more accurate than in vivo methods [2].

There have been numerous studies on single motion segment units on the lumbar spine, but it is necessary to test multi-level segments in order to understand the natural movements of the lumbar spine to take into account lordosis and adjacent level effects. Additionally, spinal constructs that involve multiple levels can really only be evaluated using whole lumbar or lumbosacral specimens. However, there is difficulty loading the in vitro lumbar spine with loads of in vivo magnitude. Without the musculature and ligaments, in vitro spines lack this stabilizing muscle stiffness making it difficult to support in vivo loads.

The pure moment methods of loading bypass this difficulty by applying no net force on the in vitro spine, but these loading conditions are not physiologically correct. The addition of follower load to pure moment methods accounts for the stiffness of the musculature by applying a load along the longitudinal axis of the specimen to increase the load carrying capacity of the in vitro spine [8]. Another methodology called eccentric loading applies some axial load but not of in vivo proportions. All of the aforementioned methods fail to include shear loads which are present in vivo. A methodology that includes axial, shear, and moment loading is necessary to realistically load in vitro specimens in order measure realistic motion. The present study introduces a new method that overcomes the loading limitations of previous in vitro methods.

With the various methods of testing in vitro, it is necessary to have a way to compare these methods. Range of motion is an easily compared kinematic characteristic. Several pure moment studies of the multi-body lumbar spine have reported intersegmental rotations, but only one follower load study was found to have reported intersegmental rotations $[2,9,10]$. With the limited amount of in vitro intersegmental rotation data sets available, it would be valuable to compare testing methodologies within the same specimen pool.

The first objective of this study was to introduce a new protocol called Combined Loading Moment that overcomes the limitations of previous methods by applying more realistic loading conditions and to compare the range of motion of this new protocol to 
those of eccentric loading and pure moment protocols within the same specimen pool. The second objective of this study was to compare these three protocols to both in vivo and in vitro data sets. 


\title{
CHAPTER 2. BACKGROUND OF THE LUMBAR SPINE
}

Located beneath the thoracic vertebrae and above the pelvis, the lumbar spine supports the majority of the weight of the body, in turn making it also the most injury prone. In order to understand the sagittal mechanics of the lumbar spine, it is important to have an adequate knowledge of the associated anatomy, basic mechanics, and range of motion.

\begin{abstract}
Anatomy
The human spine consists of twenty-four articulating vertebrae plus nine fused vertebrae. The articulating vertebrae include the seven cervical, twelve thoracic, and five lumbar vertebrae. The nine fused vertebrae are composed of the five sacral and the four coccygeal fused bodies. In lateral view, the cervical and lumbar regions exhibit inward curvature called lordosis and the thoracic and sacral regions exhibit outward or straight curvature called kyphosis. These curvatures are assumed to function in the distribution of mechanical stresses and may affect spinal mobility [1]. The amount of curvature varies from person to person, and it has been shown that the amount of lumbar lordosis in patients with low back pain may influence segmental motion, load distribution, and disc degeneration [11].

The five lumbar vertebral bodies are numbered starting from the top and are referred to as L1 through L5 as shown in Figure 2-1A. Like the other articulating vertebrae, each lumbar vertebra is made up of an anterior body and posterior elements. The lumbar anterior bodies are the largest of the articulating vertebrae in order to sustain the majority of the load on the spine. They consist of mostly of cancellous bone enclosed in a thin layer of cortical bone. The posterior elements consist of the pedicles, lamina, transverse processes, spinous processes, superior facets and inferior facets as labeled in Figure 2-1B. The pedicles act to transfer the load from the anterior body to the posterior elements. The inferior facets articulate with the superior facets of the adjacent vertebrae. These articulating facets aid in preventing hyperextension, but are less involved during flexion.

Between each vertebral body is a viscoelastic intervertebral disc that makes up about $20-33 \%$ of the entire height of the spine. Intervertebral discs carry and distribute loads, articulate rotational and translation motion, and connect the superior and inferior bodies. Each intervertebral disc is composed of an inner nucleus pulposus, an outer annulus fibrosis, and cartilaginous endplates. In addition to the intervertebral disc acting as a ligament between two vertebral bodies, the lumbar spine also encompasses six other ligaments called the anterior longitudinal ligament, posterior longitudinal ligament, ligamentum flavum, capsular ligament, interspinous ligament, and supraspinous ligament. Each of these ligaments has several functions: to allow physiologic motion and fixed posture of the vertebrae, to protect the spinal cord by limiting motion, to provide stability
\end{abstract}



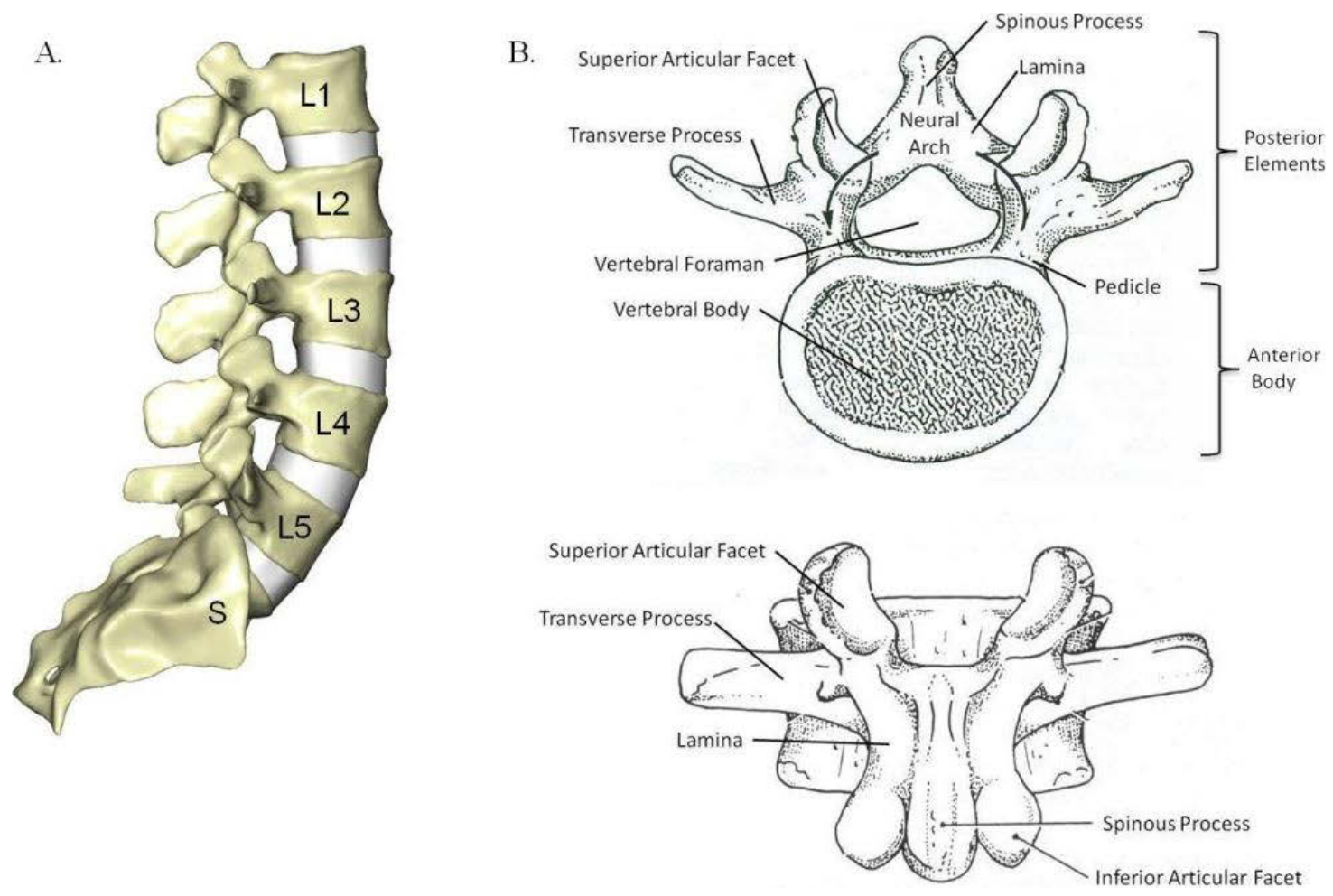

Figure 2-1. The vertebra of the lumbar spine.

A.) The lumbar spine numbered L1 to L5 from superior to inferior along with the sacrum referred to as S. B.) The elements of the lumbar vertebra. Source: Modified with permission. Adams, M.A., The Biomechanics of Back Pain. 2003, Edinburgh; New York: Churchill Livingstone. p. 19. [12]. 
in conjunction with the muscles, and to absorb large amounts of energy during traumatic situations in order bypass trauma from the spinal cord [1].

\section{Basic Mechanics}

The lumbar spine undergoes a variety of forces in vivo including the weight of the trunk and head, muscle and ligament stabilization, and external loads. Understanding the exact involvement of each of these is difficult, so this section will focus on the weight of the trunk and head. Figure 2-2 demonstrates the force of body weight on the spine. This simplified diagram shows that the body weight vector $F_{\mathrm{BW}}$ acts ventral to the spine causing a forward-bending moment during standing [13]. The magnitude of moment $\mathrm{M}$ at the L5-S disc is determined by the following equation:

$$
\mathrm{M}=\mathrm{F}_{\mathrm{BW}} \times \mathrm{d}
$$

where $\mathrm{d}$ is the distance between $\mathrm{F}_{\mathrm{BW}}$ and the center of the L5-S disc. Also acting at the center of the disc are the shear component $\mathrm{F}_{\mathrm{C}}$ and axial component $\mathrm{F}_{\mathrm{A}}$ which can be calculated by the equations:

$$
\begin{aligned}
& \mathrm{F}_{\mathrm{S}}=\mathrm{F}_{\mathrm{BW}} \times \sin \theta \\
& \mathrm{F}_{\mathrm{A}}=\mathrm{F}_{\mathrm{BW}} \times \cos \theta
\end{aligned}
$$

where $\theta$ is the angle that corresponds to the angle between $F_{\mathrm{BW}}$ and $\mathrm{F}_{\mathrm{A}} . \mathrm{F}_{\mathrm{A}}$ always acts perpendicular to the disc plane, and $\mathrm{F}_{\mathrm{S}}$ always acts along the plane of the disc. As the angle $\theta$ increases with flexion motion, the shear component of the body weight vector increases. The relationship between the moment $\mathrm{M}$ and moment arm $\mathrm{d}$ is similar. With more curvature in the spine there is a greater moment arm which produces a larger bending moment. Naturally standing the spine is still able to withstand the weight of the upper body along with external loads with the use of muscles and ligaments. Although this picture shows a very simple scenario without the complication of external loads or musculature, the shear force, axial force, and bending moment are still significant loads on the lumbar spine.

\section{Range of Motion}

Range of Motion (ROM) refers to the measured rotational displacement. The spine has six degrees of freedom as it is able to rotate sagittally, laterally, and axially. Figure 2-3 shows the degrees of freedom in both three dimensions and two dimensions. The focus of this study is sagittal rotation in two dimensions with only three degrees of freedom. The rotation about the y axis produces a sagittal moment. Translation along the $\mathrm{z}$ axis can induce axial compression, and translation along the $\mathrm{x}$ axis can induce shear loads. In order to fully understand the spine in the sagittal plane, all three degrees of freedom should be incorporated. 
A.

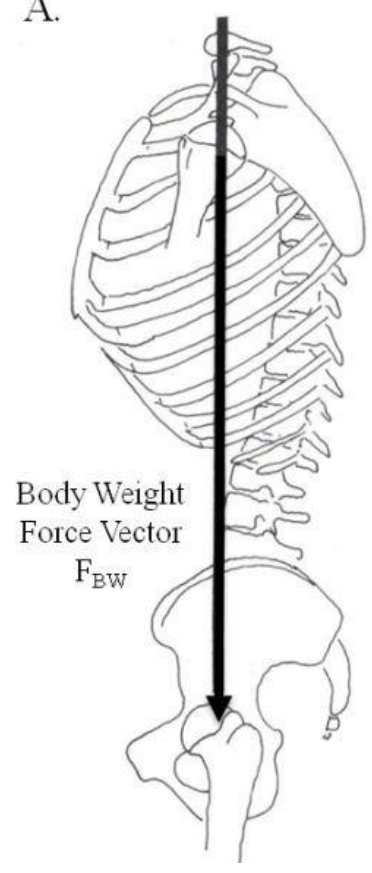

B.

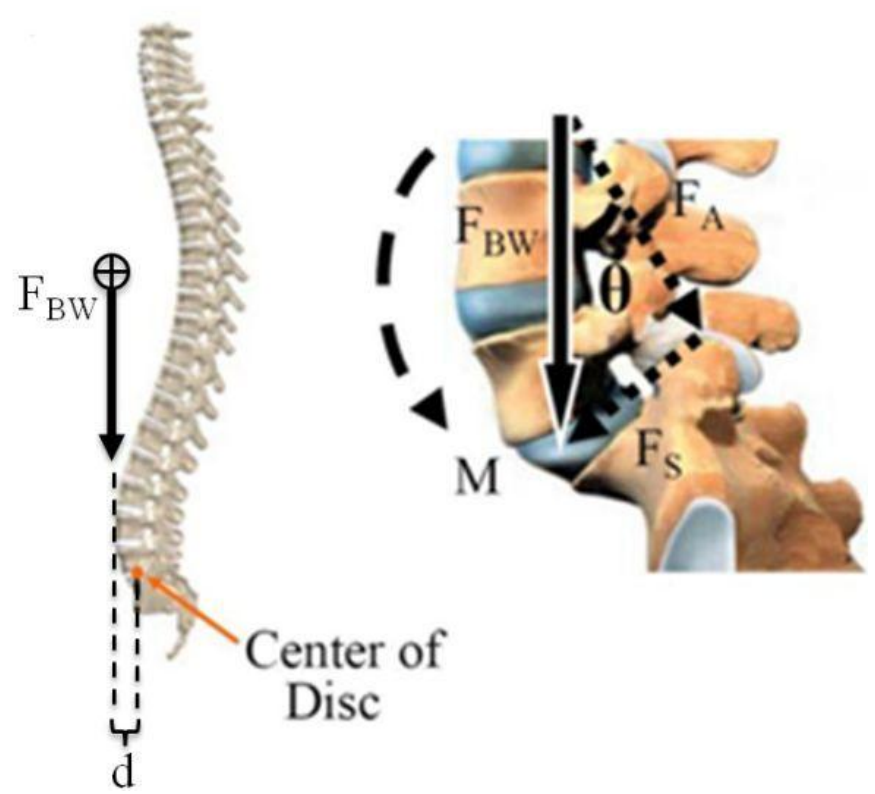

Figure 2-2. The force of body weight on the lumbar spine.

A.) The line of gravity acting on the trunk. Source: Modified with permission. Nordin, M. and V.H. Frankel, Basic Biomechanics of the Musculoskeletal System. 3rd ed. 2001, Philadelphia: Lippincott Williams \& Wilkins. p. 268. [13]. B.) The body weight vector acting at the center of the L5-S disc. Source: Modified with permission. Zufelt, N., $A$ Kinematics-Based Testing Protocol to Study the Mechanics of the Human Lumbar Spine. Master's Thesis. 2008, The University of Tennessee Health Science Center. [14]. 

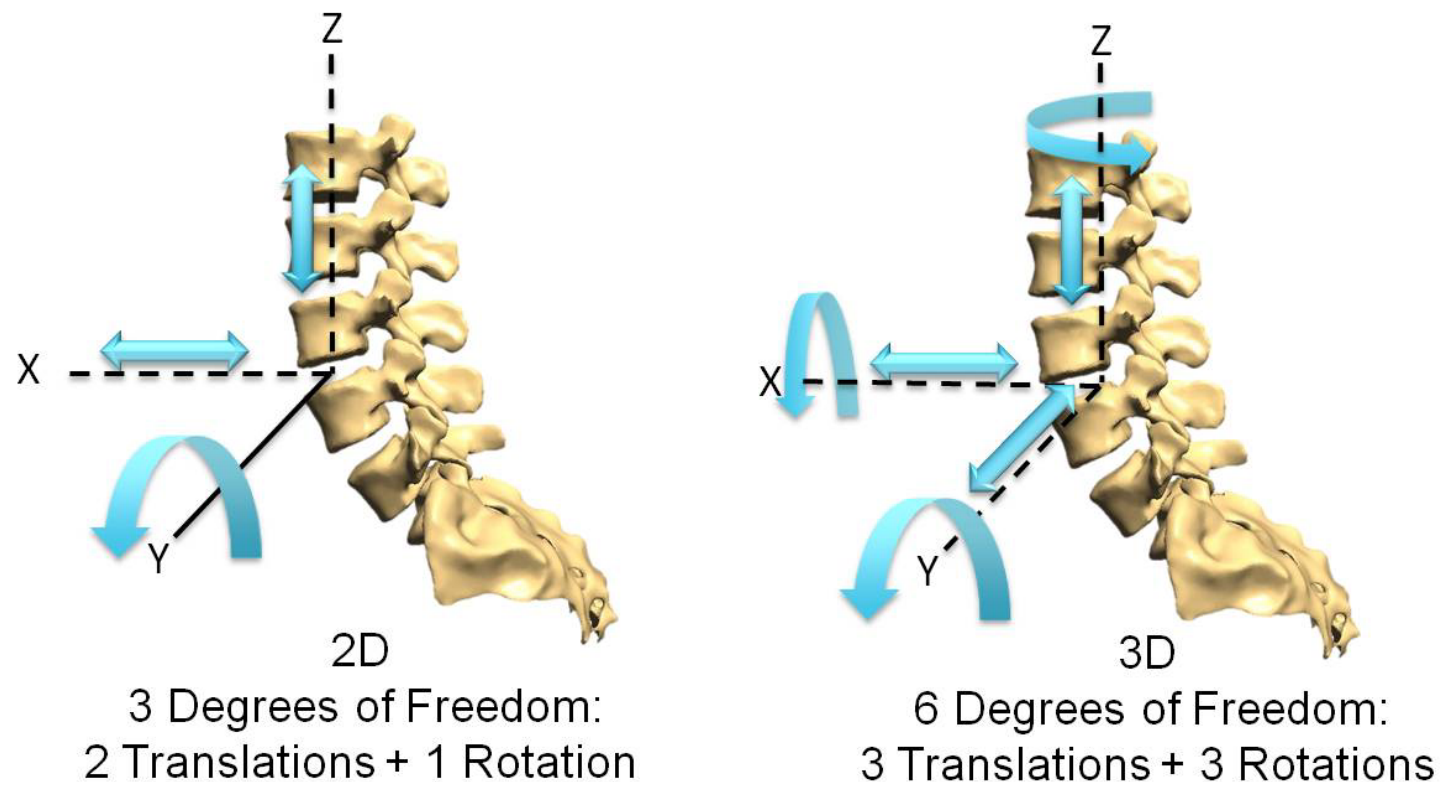

Figure 2-3. Degrees of freedom in two dimensions and three dimensions.

For complete analysis during 2D, all three degrees of freedom must be studied. 
In vitro ROM is an easily comparable kinetic characteristic among different studies. It can be measured as total global rotation of the entire lumbar segment or as individual vertebral rotations relative to the inferior segment. Since end limits and methodologies vary, the individual rotations are more easily compared between different studies when calculated as a percent of the total ROM of the entire segment. This is referred to as \%global rotation in this study. 


\section{CHAPTER 3. IN VITRO TESTING METHODOLOGIES OF THE MULTI- SEGMENT LUMBAR SPINE}

There have been numerous studies on the lumbar spine range of motion in vivo, but they are limited in the amount of information they provide $[4,6,7,15,16]$. Since most of the studies rely on lateral radiographs, they are restricted to measuring motion only in the sagittal plane. Images are taken at maximum flexion and maximum extension, and then the images are superimposed to determine the displacement between the two modes of bending at each vertebral level $[4,5,7]$. These methods fail to describe range of motion of flexion and extension separately, and there are also discrepancies between studies due to different subject positioning during imaging, active versus passive bending, and image quality. Newer methods of measuring range of motion in vivo have used video fluoroscopy and positional MRI which can distinguish between flexion and extension range of motion, but the exact loads applied to the spine still remain unknown and uncontrolled $[3,11]$.

In vitro studies provide a more controlled environment to study the physical properties of the spine. Many in vitro experiments have been conducted on single motion segment units consisting of only two vertebral bodies, but these experiments do not take into account lordosis and adjacent level effects of the whole lumbar spine. It is necessary to test multi-level specimens in order to have a more realistic representation of in vivo motion and to evaluate orthopedic devices that involve multiple levels. Load-control dominates the majority of studies done with multi-segment spines. Examples of these studies include pure moment and pure moment with the addition of follower load. Other studies involve the use of displacement control such as eccentric loading. Each of these methods and their limitations will be discussed by focusing on the three degrees of freedom involved in the sagittal plane motion. A new load control methodology that addresses the limitations of the previous methods will also be described in terms of degrees of freedom in the sagittal plane.

\section{Pure Moment}

The pure moment methodology applies a uniform pure moment to the spine. There are no axial compressive, vertical, or shear loads applied, so only one degree of freedom is studied which is the moment applied. The original methods applied this pure moment using a cable driven pulley system as shown in Figure 3-1. The two equal, opposite, and parallel forces applied vertically to the pulleys yield a resultant force of zero and generate a moment [2]. The benefit of testing multi-segmented spines under pure moment is that the moment at every level of the spine should be equal because there is no resultant force. The constant rotational load allows the structural properties of the spine to be determined. However, it has been shown that early methods of pure moment that use a fixed pulley apparatus do not maintain cable co-linearity, and the fixed pulley causes tethering of the top segment which causes erroneous reverse loading [17]. This reverse loading produces non-trivial shear loads and non-uniform loading conditions 


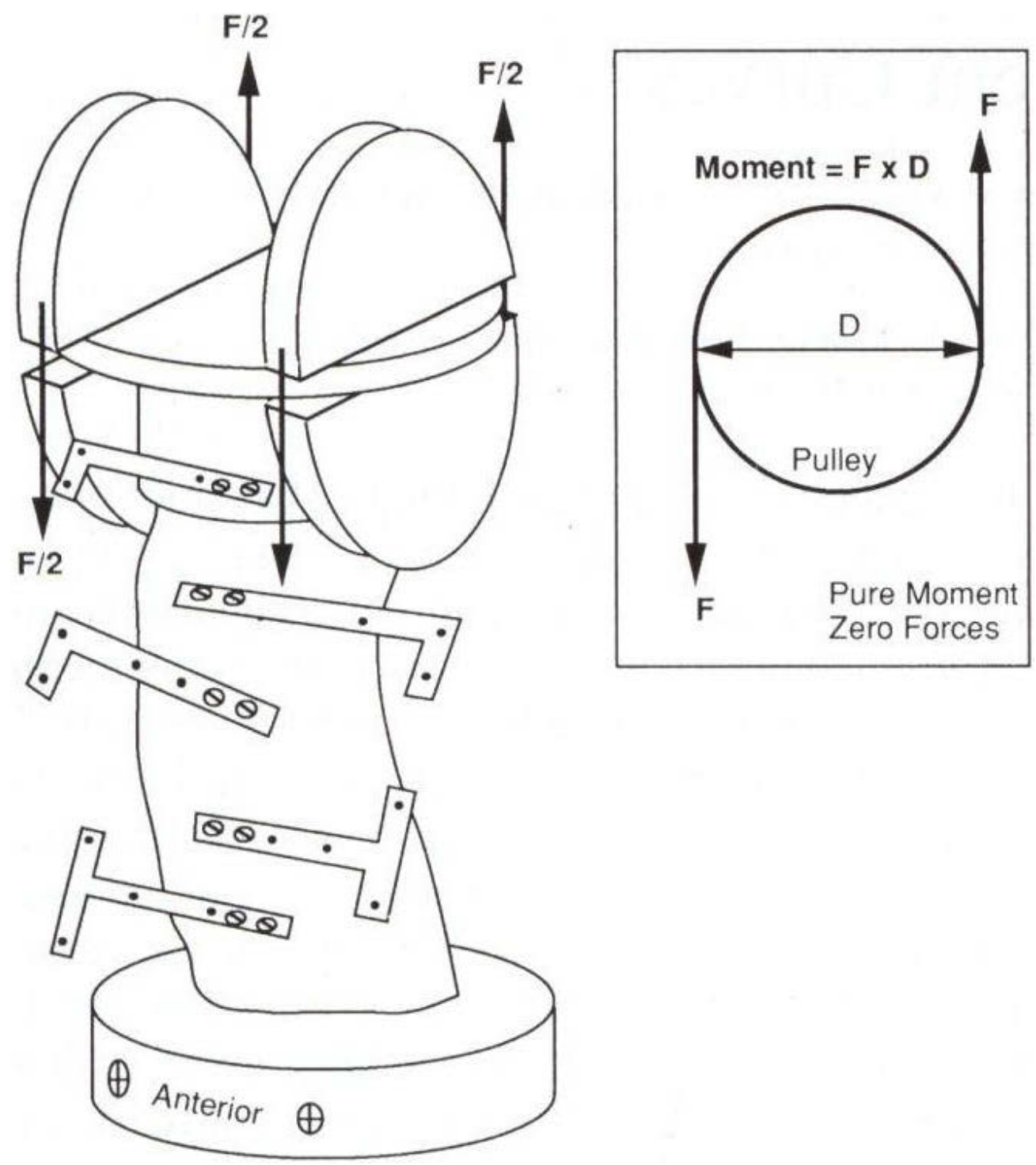

Figure 3-1. Diagram of pure moment experimental setup using pulleys.

Source: Reprinted with permission. Panjabi, M.M., et al., Mechanical behavior of the human lumbar and lumbosacral spine as shown by three-dimensional load-displacement curves. The Journal of Bone and Joint Surgery, 1994. 76(3): p. 413-24. [2]. 
along the length of the specimen which deviates from the pure moment state. Newer methods of pure moment have been developed that use a system of force controlled motors [18].

The main benefit of pure moment is the uniform loading along the length of the spine which allows the structural properties to be evaluated. Another benefit pure moment is that in vitro instability is not an issue because there are no compressive forces on the spine. The drawback of this method is that it is not physiologically correct. Lumbar spines in vivo support the weight of the upper body which applies axial and shear loads in addition to a rotational moment as explained previously in Basic Spine Mechanics. Without these physiological loads, pure moment applications are limited in helping to understand the effects of realistic spinal loads on range of motion.

\section{Addition of Follower Load}

Although pure moment allows uniform moments to be applied, it does not simulate physiological loads. The addition of follower load to pure moment applications was introduced to simulate muscle forces on the spine. These loads were applied via cables passing through the center of rotation of each disc as shown in Figure 3-2. In theory these loads would be applied at the center of rotation of each disc so as to not create any additional moments. The addition of follower load has been shown to increase the load carrying capacity of the in vitro spine [8]. The stabilization of the spine due to follower load in combination with pure moment allows the in vitro spine to withstand loads of in vivo magnitudes while still allowing physiologic mobility in flexion-extension $[9,19,20]$.

However, it is difficult to determine the exact locale of the center of rotation for each level because it has been shown the axis of rotation shifts anteriorly during flexion and posteriorly during extension [21]. The application of follower load along a path that is not tangent to the real center of rotation can induce artificial moments at each level. Although pure moment with follower load applies more realistic loading conditions than pure moment alone, the loading conditions do not include shear load. Only two degrees of freedom are incorporated; one rotation due to the moment and one translation due to the follower load.

\section{Eccentric Loading}

Unlike pure moment methods with and without follower load, eccentric loading produces a different moment at different spinal levels. A standard eccentric loading protocol was developed for multi-level spines using a single vertical actuator in a standard test frame in the UTHSC Joint Implant Biomechanics Laboratory [22-24]. With the specimen constrained at the bottom level, a lever arm is fixed at the top level as shown in Figure 3-3. The lever arm is unconstrained via a roller bearing beneath the vertical actuator in order to prevent shear forces on the spine. As the actuator moves 


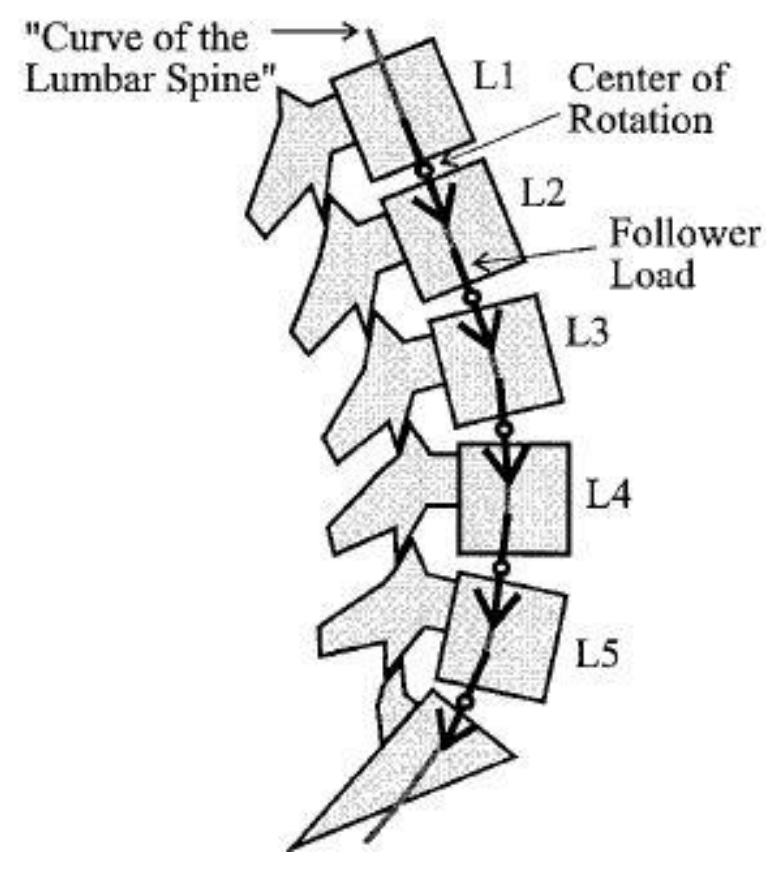

Figure 3-2. Depiction of compressive follower load.

Source: Reprinted with permission. Patwardhan, A.G., et al., A follower load increases the load-carrying capacity of the lumbar spine in compression. Spine, 1999. 24(10): $\mathrm{p}$. 1003-9. [8]. 


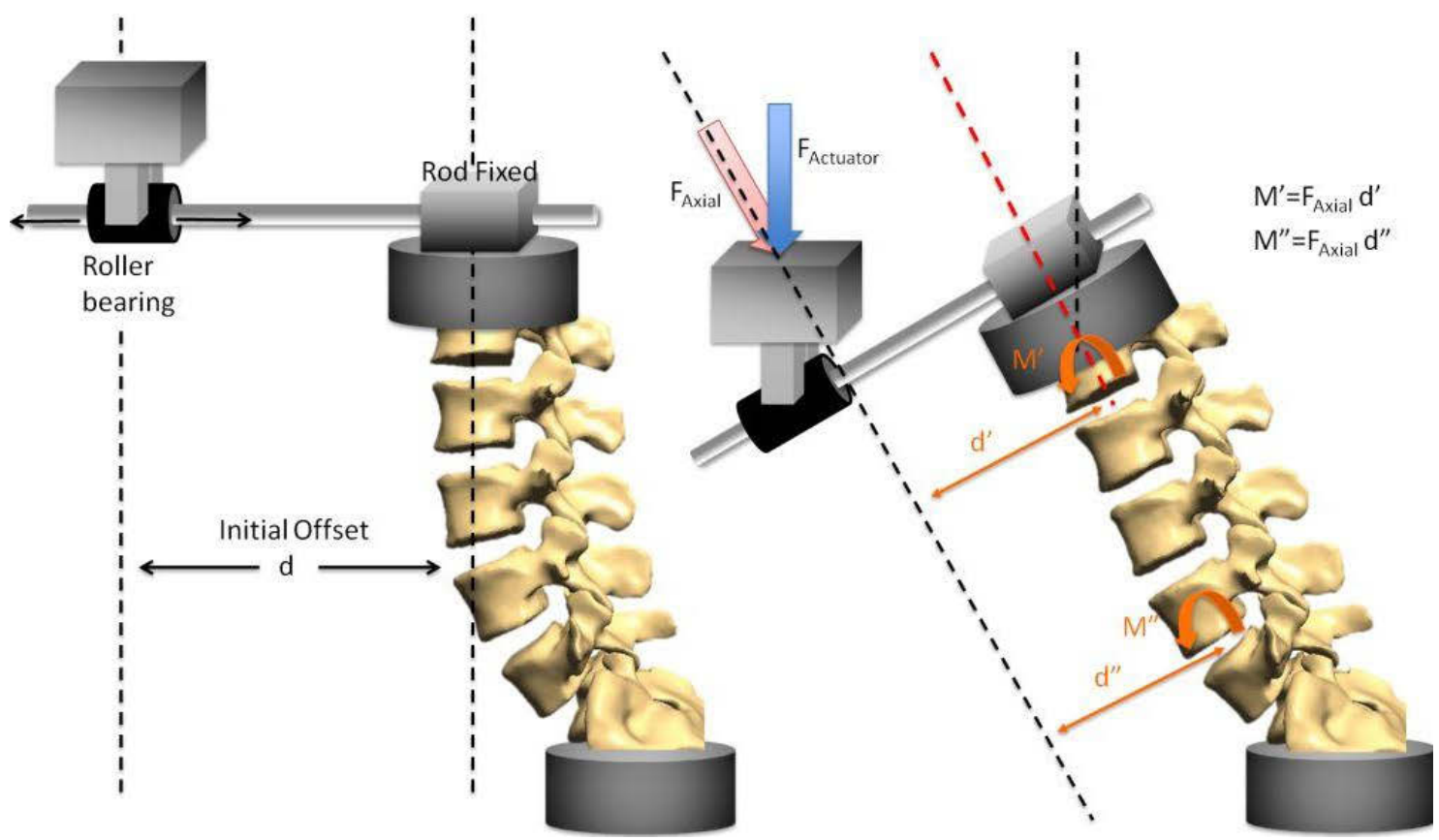

Figure 3-3. Eccentric loading diagram of setup and loads.

The initial offset decreases with flexion producing various moment values down the levels. 
down, an axial force is applied and also produces a moment on the spine. Because the specimen is not a straight column and is being flexed, the moment arm $\mathrm{d}$ varies at different levels. This variation in moment at different levels theoretically should mimic in vivo behavior better than pure moment methods [25]. However, some argue that since the different moments are not known, pure moment is more appropriate for testing spinal construct devices $[25,26]$. Opponents of this method also point out that displacement is ill-defined because the ideal location of the rotation axis remains unknown until a test is run based on an estimation of the axis [27]. To account for these difficulties in both eccentric loading and pure moment, Panjabi et al. developed a hybrid method by using a combination of moment-input methods and displacement-input methods [27]. The hybrid method still fails to incorporate all three degrees of freedom in the sagittal plane by excluding shear loads from the load application.

\section{Combined Loading Moment: A New Method}

It has been discussed that pure moment and eccentric loading methods have different advantages, but the limitation they both share is that neither introduces shear load which occurs in vivo. Combined loading moment is a new method that applies a vertical load with components of shear load and axial load with a bending moment without the use of follower load to a multi-body lumbar spine using the UTHSC Spine Robot. The new method was developed based on previous pure moment protocols used with the Spine Robot [21, 28]. These protocols were used for single motion segment units, but were developed to be used for multi-segmented spines in this study. The PM methods of the robot accounted for the pot weight of the specimen and rotated the specimen incrementally until an end limit moment was reached. At each increment the $Z$ and $\mathrm{X}$ actuator moved to maintain minimal axial and shear loads on the spine. These motions are shown in Figure 3-4. The CLM protocol applied similar methods except at each increment of rotation vertical load was maintained. The $\mathrm{Z}$ actuator moved to maintain the axial component $F_{Z}$ of the vertical force $F_{V}$ shown in Figure 3-5. The $X$ actuator did not move to maintain the shear component $F_{X}$ in order to allow the shear loads to build passively with rotation. 


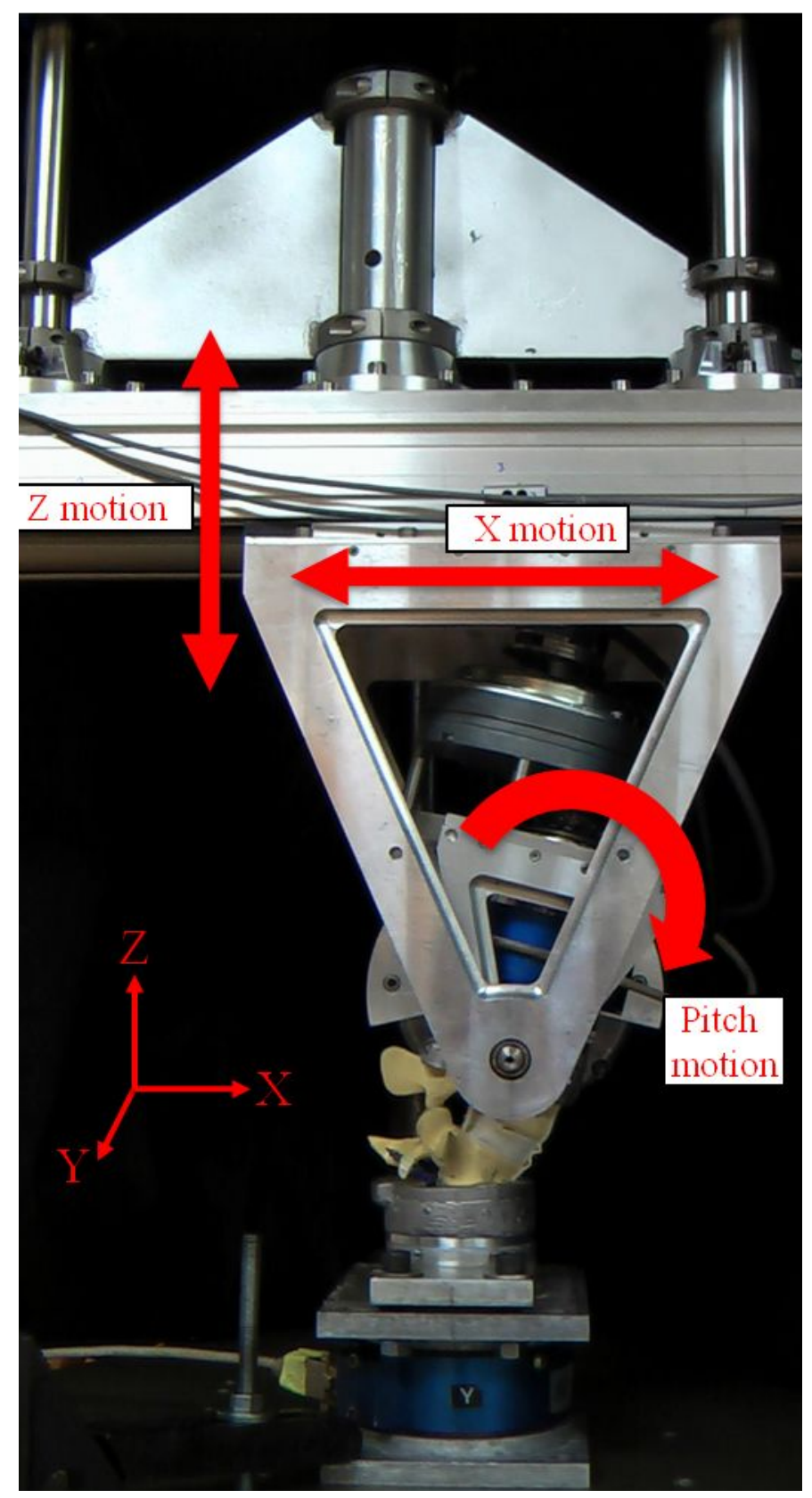

Figure 3-4. Spine robot motion during PM.

A single motion segment unit is shown in flexion during a PM protocol using the UTHSC Spine Robot. The $\mathrm{X}$ and $\mathrm{Z}$ actuator produce motion in the $\mathrm{X}$ and $\mathrm{Z}$ axis in order to minimize loads. The pitch motor rotates about the $\mathrm{Y}$ axis to generate the moment. 

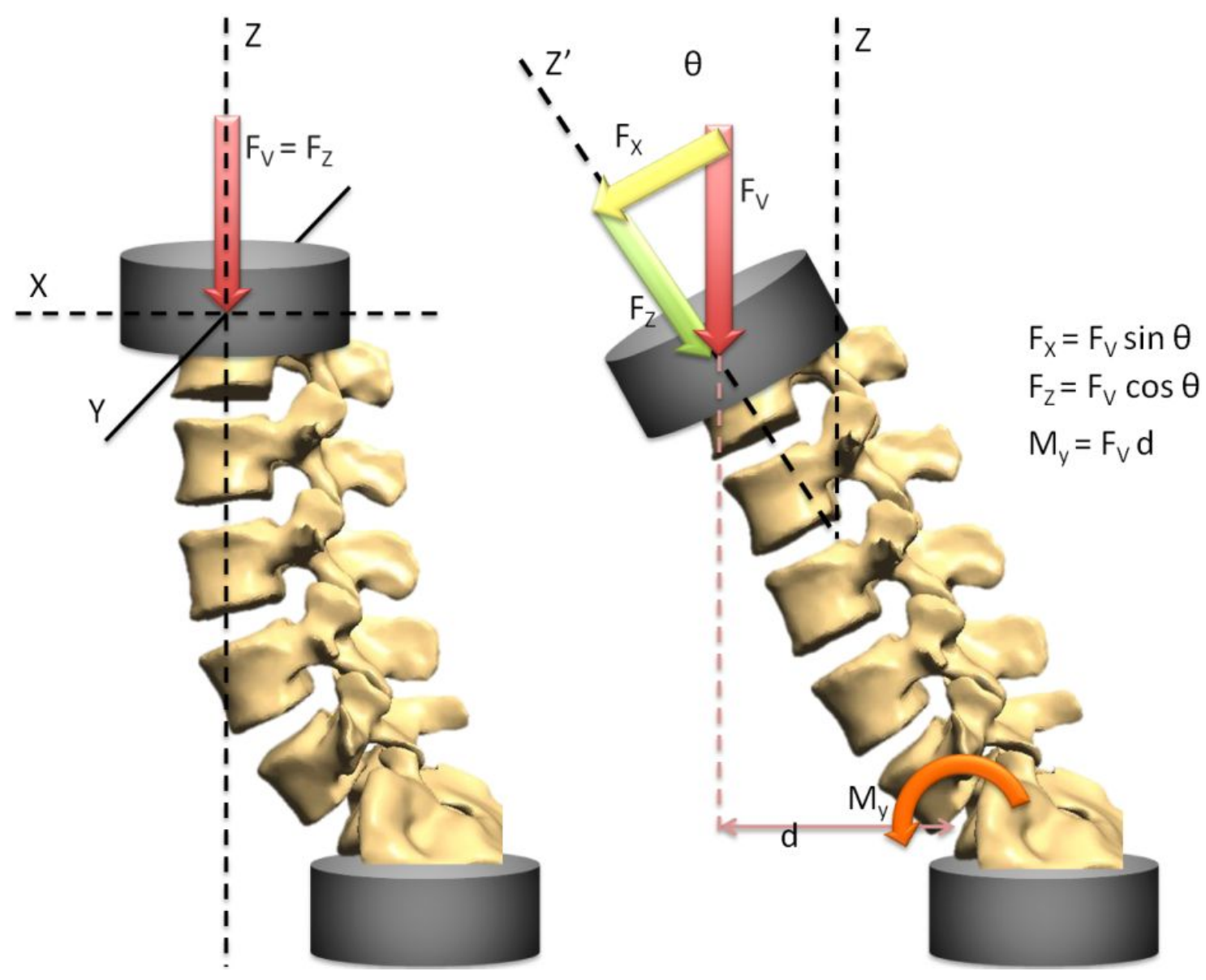

Figure 3-5. Free body diagram of the new methodology CLM.

A vertical force is applied by the $\mathrm{Z}$ actuator prior to rotation, and shear loads are minimized by moving the $\mathrm{X}$ actuator. The motions of the actuators are indicated by the dotted lines. After each rotation the vertical load $\mathrm{F}_{\mathrm{V}}$ is maintained with the axial component $\mathrm{F}_{\mathrm{Z}}$, and the shear component $\mathrm{F}_{\mathrm{X}}$ is allowed to passively increase. The moment $\mathrm{M}_{\mathrm{Y}}$ is produced by the rotation of the pitch and the vertical force. 


\section{CHAPTER 4. AN IN VITRO INVESTIGATION OF MULTI-SEGMENT LUMBAR RANGE OF MOTION USING THREE TESTING PROTOCOLS}

\section{Introduction}

The lumbar region is a recurring site of spinal disorders including low back pain which has been seen in patients with abnormal intervertebral movements [29]. Knowledge of normal intervertebral motion is needed as a standard to determine abnormal motion. In vivo methods of measuring spine motion are mostly limited to the sagittal plane in flexion plus extension based on lateral radiographs. Although in vivo methods do provide important functional information, the loads applied to the spine are unknown and the measurements can be inaccurate due to image quality. In vitro measurements allow for a more controlled load environment to determine normal mechanical and kinematic properties of the spine. There are many published biomechanical studies using single motion segment units of the lumbar spine, but studies using the whole lumbar spine are less common. It is necessary to use whole lumbar specimens in order to investigate the more natural movements that take into account lordosis and influence of adjacent levels. Of the in vitro studies done on multi-segment spines, few report the intersegmental rotations of each level.

The most common method of multi-segment testing is pure moment which produces uniform loading to each segment by applying equal and opposite forces via cable driven pulleys. The net force applied is zero, but a moment is produced. The pure moment methods allows for uniform loading conditions that are easily compared between labs using similar methods of pure moment, but these loading conditions are not realistic when compared to in vivo. Also, the accuracy of cable driven pulley methods of pure moment has been challenged by a study that found fixed pulley methods deviate from uniform loading conditions [17]. Although there have been studies using pure moment that overcome the limitations of the fixed pulley method by using robotic spine testers, few of these studies reported intersegmental rotations. In order to apply more realistic loading conditions during pure moment, follower load has been used to increase the load carrying capacity of the spine. The use of follower load simulates muscle involvement by applying a compressive load to the center of rotation of each lumbar segment. Determining the exact center of rotation for each segment is difficult since it has been shown that center of rotation shifts during different modes of bending [21]. This is a limitation of follower load because artificial moments could be produced if the path of the load is not correctly placed at the center of rotation and could artificially stabilize the specimen.

Another method of multi-segment testing is eccentric loading which applies an axial load to the top segment using a lever arm attached to a frictionless roller bearing. The axial load is applied using a vertical actuator and produces a moment without any shear load. Methods of eccentric loading produce more physiologic motion than pure moment because the loading conditions are not uniform through the column of the 
specimen. However, eccentric loading still lacks the incorporation of shear loads that are present in vivo.

Current in vitro methods are not without limitations in applying realistic loading conditions. Pure moment, follower load, and eccentric loading methods fail to incorporate shear loads that are present in everyday movement. The objective of this study was to introduce a new protocol Combined Loading Moment (CLM) that overcomes the limitations of previous in vitro methods by applying realistic loading conditions that include moment, axial, and shear loads and to compare the range of motion of this new protocol to Pure Moment (PM) and Eccentric Loading (EL) protocols within the same specimen pool. The second objective was to compare the motion profiles of these three different protocols to both in vivo data sets and previous in vitro data sets.

\section{Materials and Methods}

\section{Specimen Preparation}

Six whole lumbar spines (three females and three males ages 53 -70) were obtained and stored at $-20 \mathrm{C}$ until preparation. Antero-posterior and lateral radiographs were taken to eliminate any anatomical abnormalities. The L1 to the sacrum was excised, and the surrounding soft tissues were dissected with caution to preserve bone, discs, and spinal ligaments. The L1 body was potted with the superior end plate horizontal in a circular mold using bismuth alloy. The potted L1 body was then fixed in an alignment jig so that L1 remained horizontal and the rest of the spine was allowed to hang neutrally. The sacrum was potted in this neutral position using the same circular mold with bismuth alloy. Threaded rods were drilled into the middle of L2, L3, L4, and L5 bodies for the placement of LED targets to use with motion tracking cameras. A rod was also drilled into the L1 pot since the bismuth alloy covered the middle of the body. No rod was necessary for the sacrum since it was fixed during all motion testing. Radiographs were taken again to double check placement of rods and to compare lordosis between specimens and are found in Appendix A.

\section{Testing}

All six specimens were tested in flexion and extension using three different testing protocols: EL, PM, and CLM. The loading schematics of each protocol in flexion are shown in Figure 4-1. The same loading conditions were used during extension. All three protocols used an end limit moment of $8 \mathrm{Nm}$ or global rotation end limit of 30 degrees in flexion and 25 degrees in extension. A motion tracking camera system was used during all three protocols to measure the individual rotations for each vertebral body. 


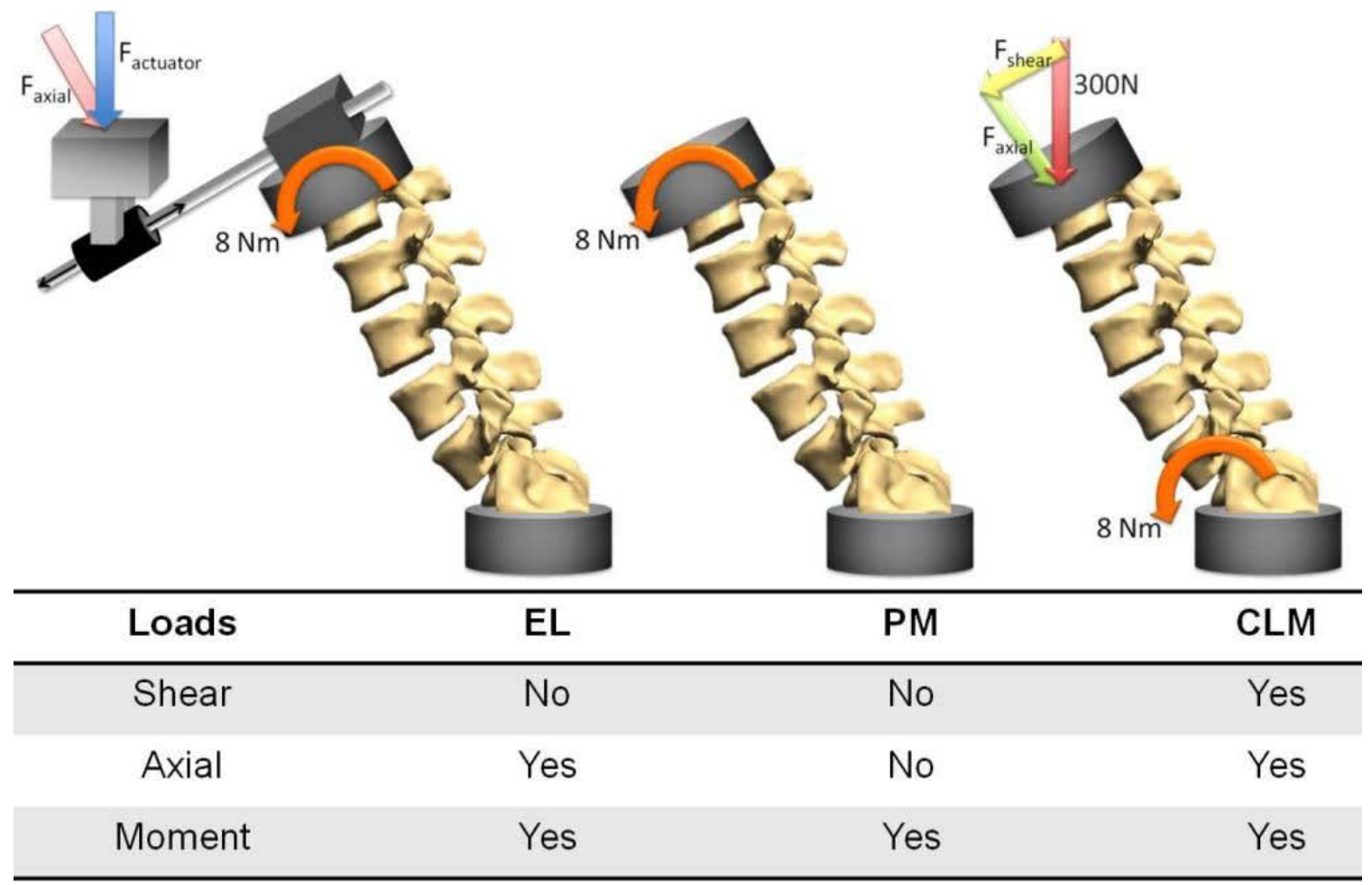

Figure 4-1. Schematic drawing and summary of loads for each protocol. 


\section{Eccentric Loading}

The specimens were first tested using the EL loading conditions using a singleactuator, biomechanical test frame $[22,23]$. Specimens were positioned with a $200 \mathrm{~mm}$ distance between the middle of L1 and the actuator load axis as shown in Figure 4-2. The sacrum was totally constrained. A metal rod was secured to the L1 fixture, but was still free to translate under the vertical actuator via a roller bearing. This eliminated most shear load. A rotary variable differential transducer (RVDT) was attached to the roller bearing and measured global rotation. A linear variable differential transducer (LVDT) measured the change in the distance between the L1 body and actuator axis as the actuator moved down. A load cell measured the axial load applied by the vertical actuator.

The LED rotations, global rotation, change in moment arm distance, and axial load were all monitored using a LABVIEW VI. The moment was calculated within the VI using the LVDT value subtracted from the initial offset of $200 \mathrm{~mm}$ and the recorded axial loads. The vertical actuator was lowered in millimeter increments until the moment end limit or global rotation end limit was met. Once the correct vertical distance was determined, the actuator repeated this same distance over five cycles. The third cycle was used for load and motion analysis.

\section{Pure Moment}

The specimens were tested first in PM using the UTHSC Spine Robot with the same initial setup shown in Figure 4-3 [28]. The specimen was positioned in the robot frame with a 100lb six axis load cell above L1 and a 1000lb six axis load cell under the sacrum. A longer mounting fixture was used to accommodate the protruding rods within the robot gimbal. The weight of the fixture was zeroed out prior to loading of the specimen. Pot weight for each specimen was zeroed out within the program so that the specimen experienced no loads. The sacrum and bottom load cell remained totally constrained throughout testing.

During the PM protocol, the specimens were incrementally rotated 0.25 degrees until an end limit was reached using the top load cell. After each increment the vertical and horizontal actuator moved in order to maintain minimal axial and shear loads. Once an end limit was reached, the same motion was repeated and recorded using the camera motion tracking system with the same LABVIEW VI used during EL.

\section{Combined Loading Moment}

With the same initial positioning as the PM protocol shown in Figure 4-3, the CLM protocol first applied a $300 \mathrm{~N}$ vertical load. Shear loads were initially minimized by moving the horizontal actuator before incremental rotation began. Camera motion data was collected during load application and shear minimization. Axial and shear loads 


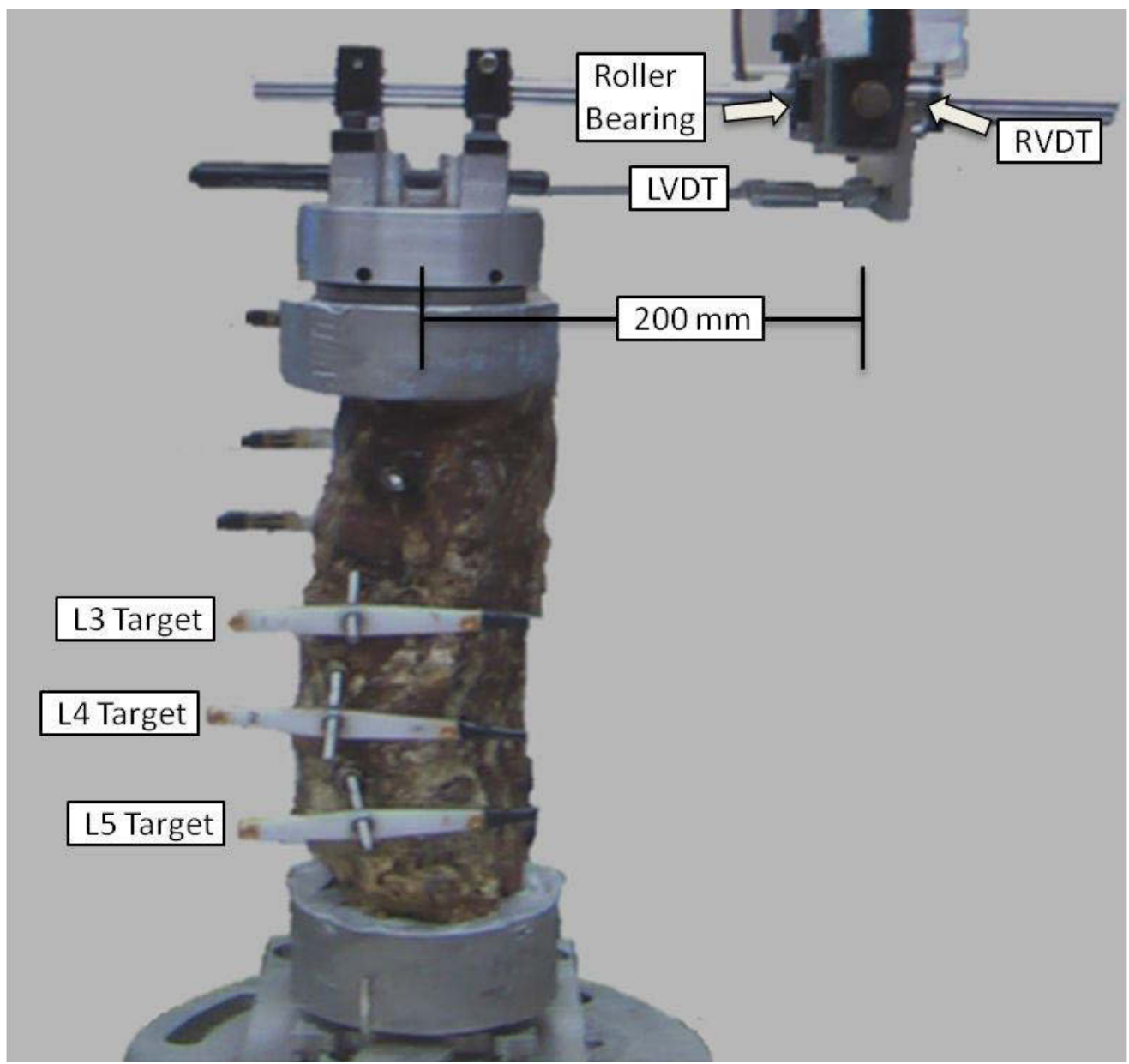

Figure 4-2. Test setup for EL protocol.

Specimen is shown in extension. The vertical actuator and load cell are not shown. 


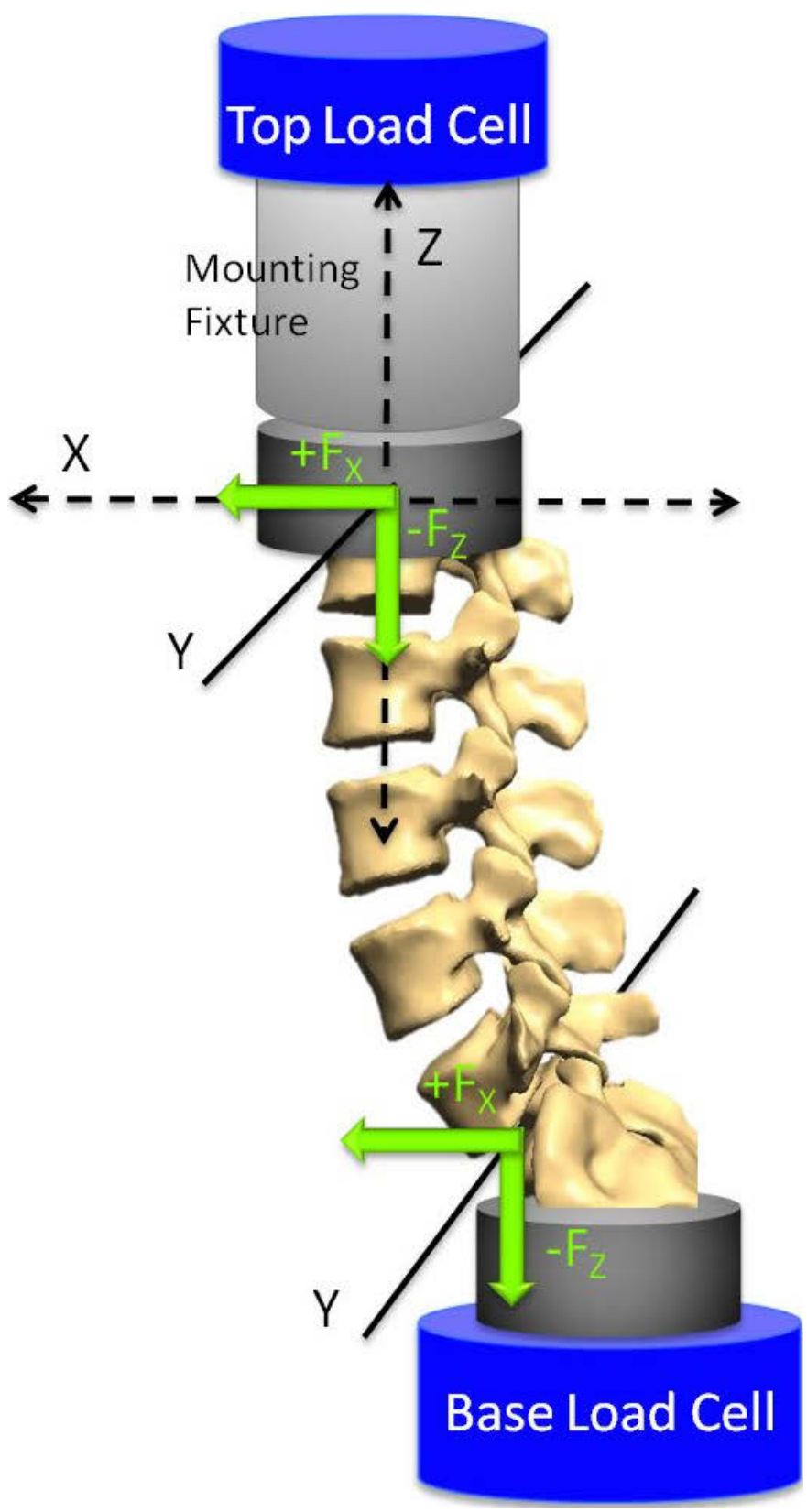

Figure 4-3. Test setup for PM and CLM with force frame transformations for each load cell.

The top load cell measured loads at L1, and the bottom load cell measured loads at the L5-S disc space. Dotted lines represent motion in the $\mathrm{x}$ and $\mathrm{z}$ planes. 
were measured using the top load cell, and the end limit moment was monitored using the bottom load cell. Specimens were again incrementally rotated 0.25 degrees until end limits were reached. At each increment the vertical load was maintained at $300 \mathrm{~N}$, and the shear load was not maintained but allowed to passively build. Once end limits were reached, the same motion was repeated in order to obtain camera motion data.

Statistical analysis was performed using SigmaStat 3.5 (Systat Software Inc., San Jose, CA). Assuming normality, global rotations and intersegmental rotations were compared using One Way Repeated Measures ANOVA with Student-Newman-Keuls Method. Significant differences shown in Figure 4-4 have p values less than 0.05. Flexibility curves were also generated for each specimen and are found in Appendix B.

\section{Results}

During flexion, EL average global rotations were significantly less than PM and CLM averages. During extension, PM average global rotations were significantly greater than both EL and CLM averages. When analyzed as total range of motion, EL global rotation was significantly less than both PM and CLM, but no significance difference was found between the two robot protocols. Overall, PM showed the greatest amount of rotation.

The flexion-extension motion profile showed significant differences at the L3-L4 and L4-L5 levels. During CLM, L3-L4 contributed significantly more motion than during PM and EL. The L4-L5 level contributed significantly less motion during EL than CLM and PM. When separated into flexion only, L3-L4 and L4-L5 still showed significant variation as well as at the L5-S level. The extension motion profiles showed significant variation at every level except L3-L4.

Table 4-1 lists the average rotations during flexion and extension and as total ROM. Intersegmental rotations showed significant differences at the L3-L4 and L4-L5 levels in flexion, extension, and total range of motion. At the L3-L4 level in flexion all the three protocols exhibited significant differences, but in extension only CLM and PM differed significantly. At the L4-L5 level in flexion PM was significantly greater, and in extension EL was significantly less than the other two protocols. When analyzed as total range of motion, EL was significantly less than the other two protocols at both the L3-L4 and L4-L5 levels. With such high standard deviations at these levels during CLM and PM, Grubb's test was used to detect any outliers, but none were found.

\section{Discussion}

In the present study, PM exhibited the most global rotation in flexion, extension, and total ROM. With the absence of loads on the spine, PM allows for greater ROM, which is most likely due to the facets being lifted to disengage allowing more unrestricted motion. Axial loading may limit overall motion since EL showed significantly less 
Average Global Rotation at $8 \mathrm{Nm}$

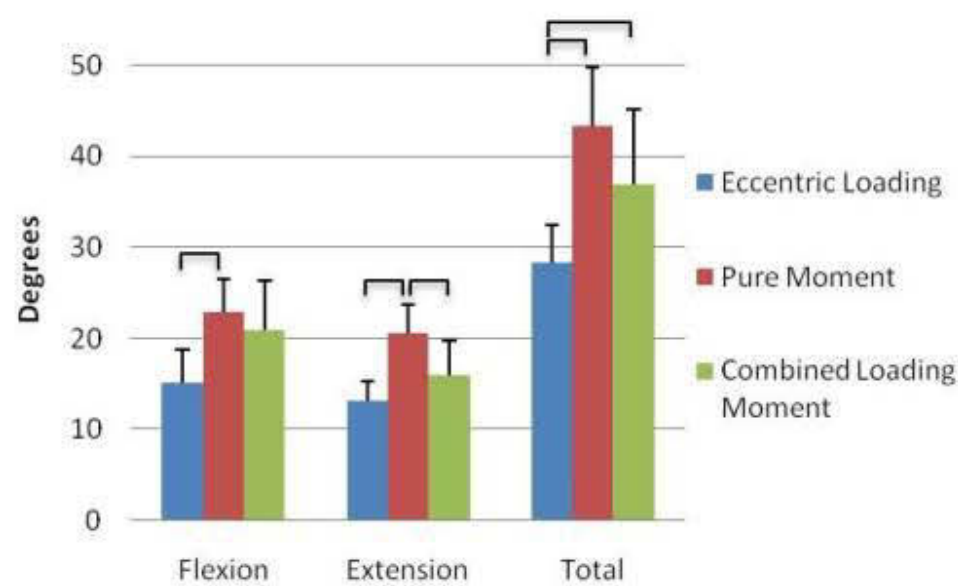

\%Global Rotation in Flexion $8 \mathrm{Nm}$

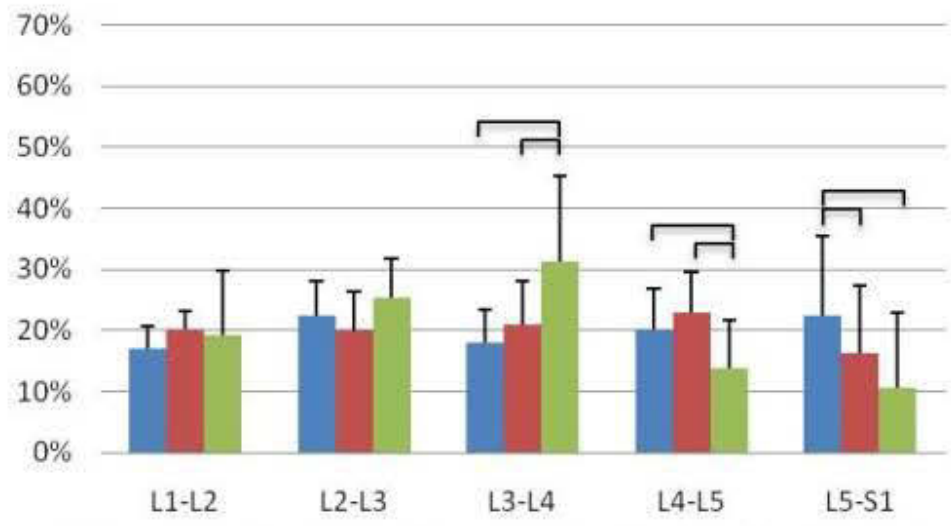

=Eccentric Loading $=$ Pure Moment $=$ Combined Loading Moment
\%Global Rotation in Flexion + Extension

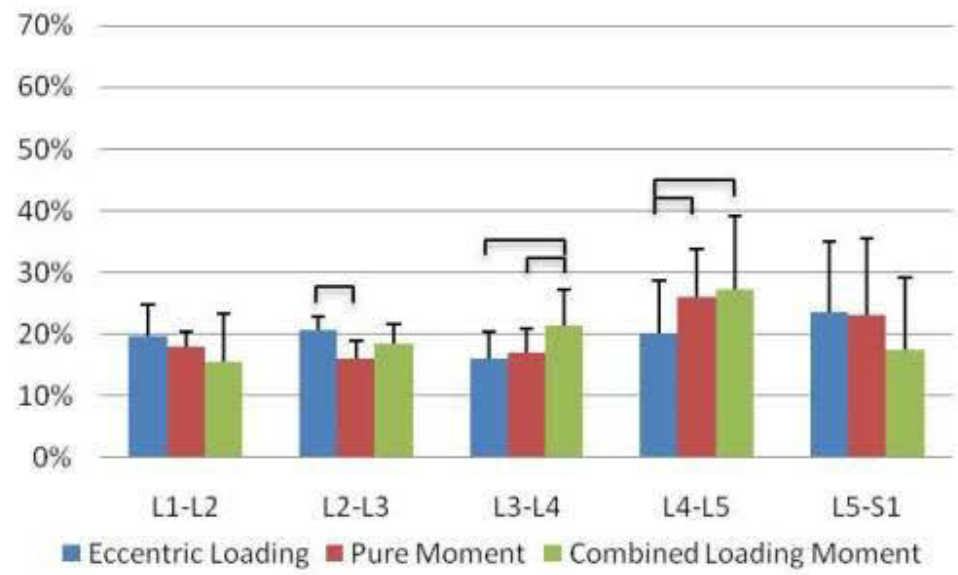

\%Global Rotation in Extension $8 \mathrm{Nm}$

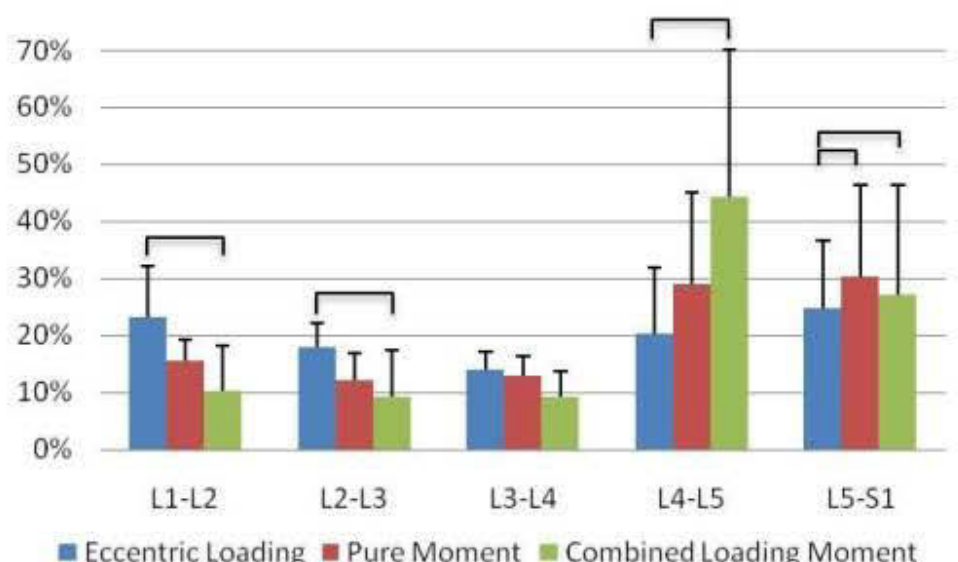

Figure 4-4. Comparisons of average global rotations and \%global motion profiles. 
Table 4-1. Average intersegmental and global rotations in degrees and standard deviations.

\begin{tabular}{|c|c|c|c|c|c|c|c|c|c|c|c|c|c|}
\hline $\begin{array}{l}\text { Mode of } \\
\text { Bending }\end{array}$ & Protocol & L1-L2 & $\mathrm{SD}$ & L2-L3 & $\mathrm{SD}$ & L3-L4 & $\mathrm{SD}$ & L4-L5 & $\mathrm{SD}$ & L5-S1 & $\mathrm{SD}$ & Global & $\mathrm{SD}$ \\
\hline \multirow{3}{*}{ 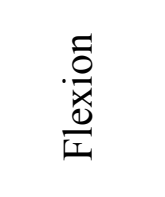 } & EL & 2.64 & 0.95 & 3.45 & 1.34 & 2.81 & 1.17 & 3.07 & 1.27 & 3.20 & 1.68 & 15.17 & 3.53 \\
\hline & PM & 4.17 & 0.44 & 4.28 & 1.61 & 4.37 & 1.59 & 4.90 & 1.98 & 3.40 & 2.08 & 22.88 & 3.56 \\
\hline & CLM & 4.50 & 2.94 & 5.51 & 2.27 & 6.28 & 1.79 & 2.87 & 1.65 & 2.28 & 2.54 & 20.96 & 5.37 \\
\hline \multirow{3}{*}{ 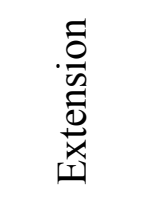 } & EL & 3.01 & 1.25 & 2.38 & 0.82 & 1.81 & 0.53 & 2.66 & 1.78 & 3.16 & 1.47 & 13.14 & 2.14 \\
\hline & $\mathrm{PM}$ & 2.97 & 0.34 & 2.32 & 0.77 & 2.48 & 0.44 & 5.69 & 3.12 & 6.17 & 3.67 & 20.51 & 3.14 \\
\hline & CLM & 1.54 & 0.98 & 1.32 & 0.97 & 1.40 & 0.66 & 6.58 & 3.25 & 4.40 & 3.60 & 15.88 & 3.92 \\
\hline \multirow{3}{*}{$\stackrel{\frac{\pi}{0}}{0}$} & EL & 5.65 & 1.98 & 5.83 & 1.17 & 4.62 & 1.58 & 5.73 & 2.69 & 6.37 & 2.66 & 28.31 & 4.21 \\
\hline & $\mathrm{PM}$ & 7.14 & 0.47 & 6.61 & 1.65 & 6.85 & 1.65 & 10.59 & 3.29 & 9.57 & 5.31 & 43.39 & 6.46 \\
\hline & CLM & 6.03 & 3.27 & 6.83 & 2.12 & 7.68 & 1.96 & 9.45 & 2.50 & 6.68 & 4.46 & 36.84 & 8.25 \\
\hline
\end{tabular}


motion than PM, but CLM showed more rotation than EL despite the application of much more axial load during CLM compared to EL. CLM also allowed passive shear to build while EL allowed only minimal shear loads. Thus, the greater ROM seen for CLM could possibly be due to the introduction of shear loads driving more motion especially during flexion. Figure 4-5 reveals the relationship between shear and rotation in both modes of bending for one specimen during CLM. In extension, the shear loads appear to increase with rotation only up to a point which could be the engaging of the facets causing shear to build in the opposite direction preventing further rotation. The effects of axial and shear loads on overall rotation were distinguished based on the differences in loading conditions among the three protocols.

\section{In Vivo Comparison}

Figure 4-6 shows the flexion plus extension motion profiles of five different data sets taken from in vivo along with the three profiles of the present study $[1,3-5,7]$. At the cephalic lumbar levels EL was greater than all the in vivo studies, but at the next two levels EL was less than in vivo. The EL pattern was motion similar to Pearcy et al. except at the L5-S level [7]. The motion profile for PM was also closest in resemblance to Pearcy et al. except at the L2-L3 level [7]. CLM followed the same trend as Ahmadi et al. and Dvorak et al., but with greater motion at the cephalic levels and less motion at the L5-S level [3, 4]. Neither EL nor PM shared the same trend at every level as any of the in vivo studies. This could be due to the unrealistic loads being applied. Since CLM showed a similar trend to some of the in vivo studies, this load scenario may be the most realistic; although, the variation seen among the different in vivo studies suggests that comparisons to in vivo are not substantial for evaluation of the present study. Some in vitro studies may even be more accurate than in vivo studies, and it is necessary to compare the present study to previous in vitro studies as well [2].

\section{In Vitro Comparison}

Numerous labs have studied the in vitro lumbar spine using methods of pure moment, pure moment with follower load, and eccentric loading, but only a few have reported the intersegmental rotations for every lumbar level. Figure 4-7 shows flexionextension motion profiles from Wong using eccentric loading, Yamamoto and Panjabi using pure moment, and Renner using a pure moment with follower load along with the motion profiles of the present study and an average of the five in vivo studies previously mentioned $[2,9,10,30]$. The average of in vivo values was used as a reference since there was so much variation among the different in vivo studies $[1,3-5,7]$. The eccentric loading profiles of the present study and Wong are very similar except at the L2-L3 level and L3-L4 level where the levels appear to be transposed. Neither study compared very well to the in vivo average because both studies showed decreases in middle levels of the motion profile where as the in vivo motion profile didn't show a decrease in rotation until the L5-S level. 


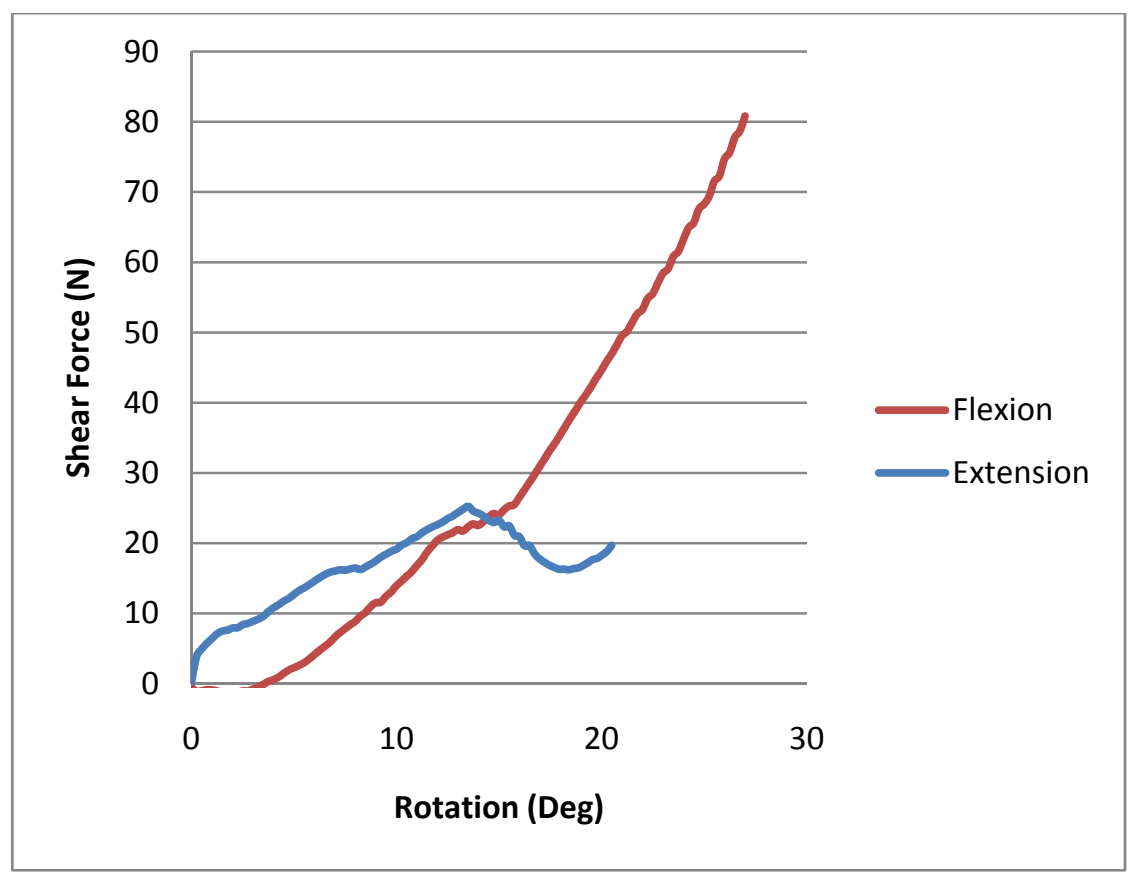

Figure 4-5. The relationship between shear force and rotation for one specimen during flexion and extension under Combined Loading Moment. 


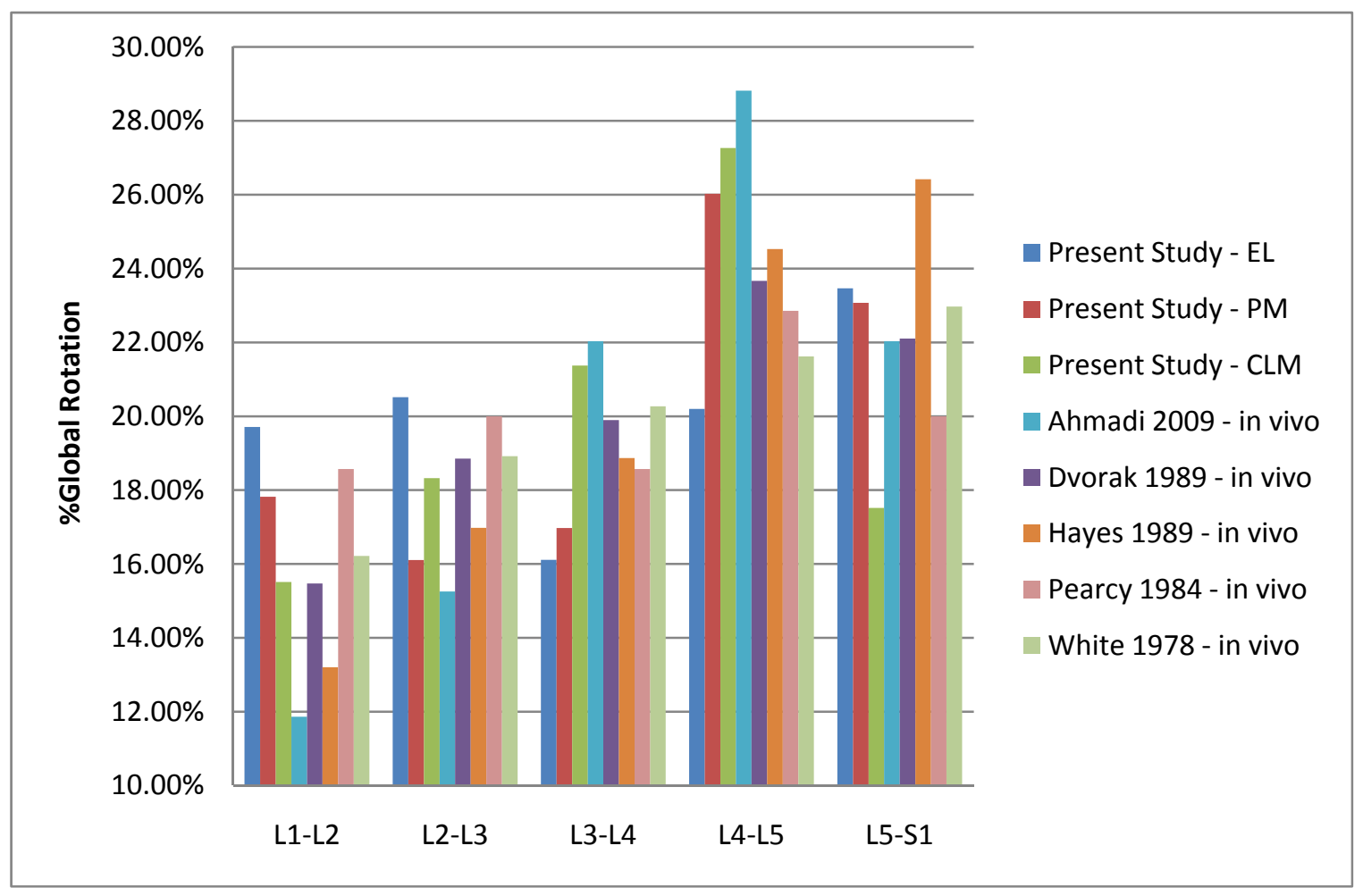

Figure 4-6. Comparison of the present study to previous in vivo studies. Intersegmental rotations reported by Ahmadi, Dvorak, Hayes, Pearcy, and White were used to calculate \%global rotations. Sources: Ahmadi, A., et al., Kinematic analysis of dynamic lumbar motion in patients with lumbar segmental instability using digital videofluoroscopy. European Spine Journal, 2009. 18(11): p. 1677-85. Dvorak, J., et al., Functional radiographic diagnosis of the lumbar spine. Flexion-extension and lateral bending. Spine, 1991. 16(5): p. 562-71. Hayes, M.A., et al., Roentgenographic evaluation of lumbar spine flexion-extension in asymptomatic individuals. Spine, 1989. 14(3): p. 327-31. Pearcy, M., I. Portek, and J. Shepherd, Three-dimensional x-ray analysis of normal movement in the lumbar spine. Spine, 1984. 9(3): p. 294-7. White, A.A. and M.M. Panjabi, Clinical Biomechanics of the Spine. 2nd ed. 1990, Philadelphia: Lippincott. p. 86-113. [1, 3-5, 7]. 


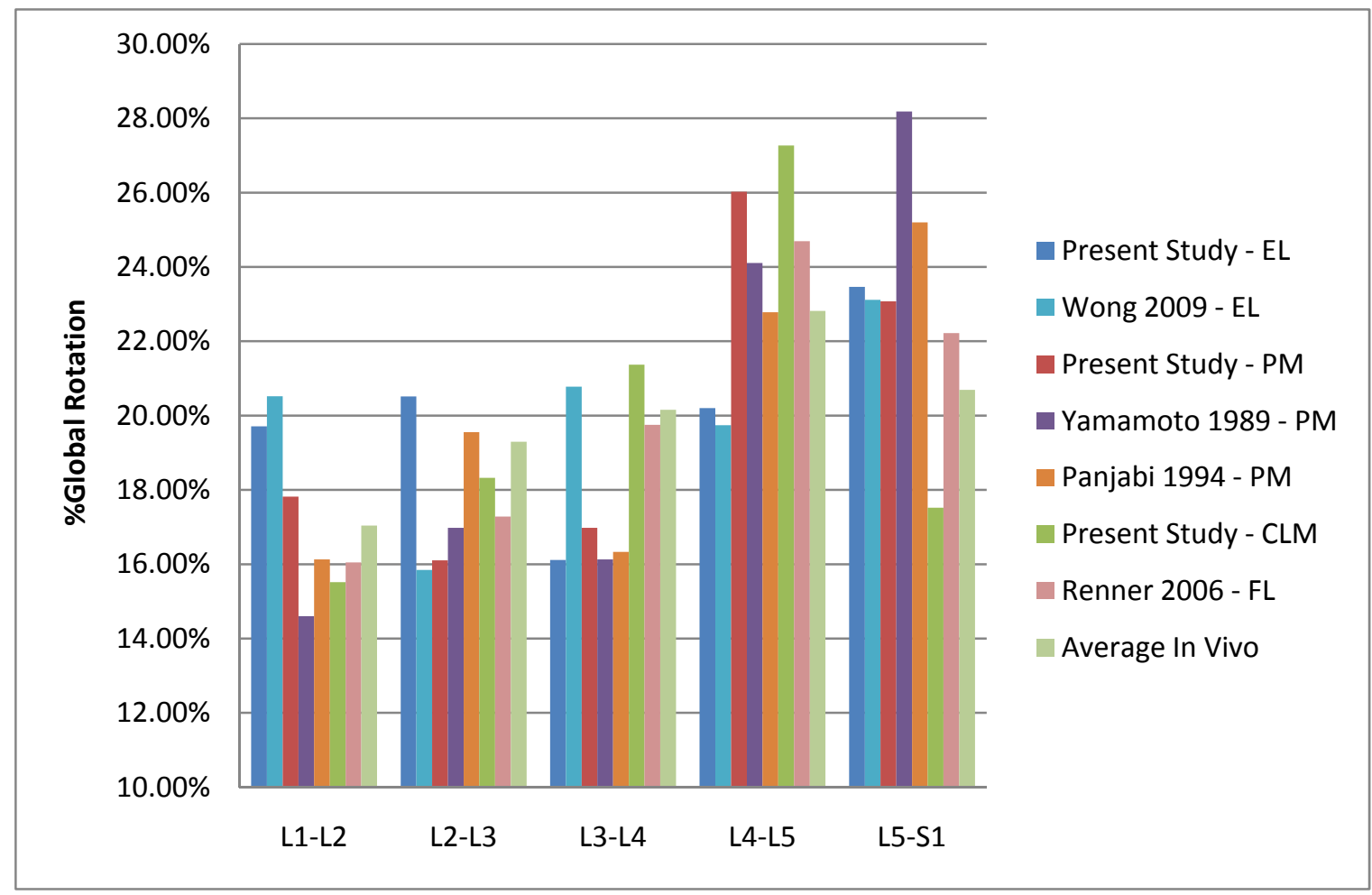

Figure 4-7. Comparison of the present study to previous in vitro studies and the average of several in vivo studies.

Intersegmental rotations reported by Wong, Yamamoto, Panjabi, and Renner were used to calculate $\%$ global rotations. An average of $\%$ global rotations for in vivo studies was calculated based on the rotations reported by Ahmadi, Dvorak, Hayes, Pearcy, and White. Sources: Wong, P., Biomechanical Comparison of Lumbar Disc Replacement. Master's Thesis. 2009, University of Tennessee Health Science Center. Yamamoto, I., et al., Three-dimensional movements of the whole lumbar spine and lumbosacral joint. Spine, 1989. 14(11): p. 1256-60. Panjabi, M.M., et al., Mechanical behavior of the human lumbar and lumbosacral spine as shown by three-dimensional load-displacement curves. The Journal of Bone and Joint Surgery, 1994. 76(3): p. 413-24. Renner, S.M., et al., Novel model to analyze the effect of a large compressive follower pre-load on range of motions in a lumbar spine. Journal of Biomechanics, 2007. 40(6): p. 1326-32. Ahmadi, A., et al., Kinematic analysis of dynamic lumbar motion in patients with lumbar segmental instability using digital videofluoroscopy. European Spine Journal, 2009.

18(11): p. 1677-85. Dvorak, J., et al., Functional radiographic diagnosis of the lumbar spine. Flexion-extension and lateral bending. Spine, 1991. 16(5): p. 562-71. Hayes, M.A., et al., Roentgenographic evaluation of lumbar spine flexion-extension in asymptomatic individuals. Spine, 1989. 14(3): p. 327-31. Pearcy, M., I. Portek, and J. Shepherd, Three-dimensional $x$-ray analysis of normal movement in the lumbar spine. Spine, 1984. 9(3): p. 294-7. White, A.A. and M.M. Panjabi, Clinical Biomechanics of the Spine. 2nd ed. 1990, Philadelphia: Lippincott. p. 86-113. [1-5, 7, 9, 10, 30]. 
Both Yamamoto and Panjabi's pure moment motion profiles showed the greatest motion to be at the L5-S level, unlike the present study and the in vivo average which both showed the greatest rotation to be at the L4-L5 level and a decrease in rotation at the L5-S level $[2,10]$. The pure moment method of the present study overcomes the limitations of the previous pulley methods by minimizing shear and axial loads while applying a constant moment using horizontal, vertical, and rotational actuators of the spine robot. The shear and axial loads were confirmed to be minimal using load cells placed above and below the specimen, whereas Yamamoto and Panjabi do not describe the placement of a load cell to confirm the uniform loading of pure moment $[2,10]$. Without the limitations of previous pure moment methods, the present study PM protocol is more accurate and also compares favorably with in vivo motion profiles.

The combined loading moment protocol is most easily compared to pure moment with follower load studies because both apply loads of in vivo magnitudes. Only one in vitro study using follower load was found in the literature that reported intersegmental rotations [9]. Despite differences in loading, the present study CLM motion profile was similar to the trend of Renner, and both compared favorably to the in vivo average. CLM showed the largest deviation from the in vivo average alternately at the last two levels, less at L5-S and more at the L4-L5. Upon comparison of the motion profiles of the 6 individual specimens of the present study in CLM, it was found that two specimens in particular showed very little motion at the sacral level and compensated with increased motion at the L4-L5 level. These two specimens were also the most lordotic of the specimens with L5-S disc angles greater than 40 degrees. A recent in vivo study using functional MRI determined that patients with a higher angle of sagittal lordosis tended to have less motion at the L5-S level [11]. This study compared the motion profiles of patients with straight, normal, and hyperlordotic spines to explain how sagittal lordosis may influence segmental motion and load distribution, but since this study only included patients with low back pain the motion profiles were not used for in vivo comparison.

\section{Limitations}

One of the limitations of the present study is that specimens were not selected based on varying amounts of lordosis and with a relatively small sample size of only six specimens, high standard deviations were seen during the CLM protocol because of the more realistic loading conditions. The addition of more specimens to this study would help create a more accurate motion profile for the CLM method. Another limitation of this study is that the eccentric loading protocol was not tested using the Spine Robot, and shear loads were not measured to verify their absence. Also, the present study only evaluated flexion and extension modes of bending. Future studies using the CLM protocol should incorporate lateral bending to further dispute the necessity of follower load to apply loads of in vivo magnitude. The comparisons to in vivo and in vitro studies were also limited because separate flexion and extension motion profiles were rarely reported, so comparisons were only made to total flexion plus extension motion profiles. 


\section{CHAPTER 5. DISCUSSION}

In the previous chapter, the effects of axial and shear loads on overall rotation were distinguished based on the differences in loading conditions among the three protocols. In addition to overall rotation, the three protocols also showed significant variation in the motion profiles at multiple levels when analyzed as flexion and extension separately and as total range of motion flexion plus extension. Larger standard deviations appeared when motion profiles were displayed separately as flexion and extension indicating that axial and shear loading can have alternate effects during different modes of bending. Some specimens might rotate more at certain levels during flexion but rotate less at the same levels during extension, but when compared as total flexion plus extension less variation is seen. An in vivo study by Pearcy et al. also reported contrasting behavior at the L5-S level during flexion and extension and suggested it could be due to the lordosis of the specimen [7]. The statistical differences seen at multiple levels show that variability in load application and lordosis of the specimens significantly affects motion profiles. In order to determine which load application is most realistic or physiologic, it is necessary to compare to both in vivo and in vitro motion profiles, but there are many factors that could contribute to the inconsistencies seen among published data sets. Table 5-1 shows the flexion plus extension absolute range of motion reported for each level by the in vivo and in vitro studies discussed in the previous chapter.

There is much variation among the in vivo profiles which could be due to a number of factors based on the subject group alone including sample size, age, gender, height, weight, overall health, and history of back pain. In addition to factors based on the subject, test method, initial positioning, active versus passive bending, length of test time, and computational accuracy and repeatability could also affect the differences seen between each study. Ahmadi et al. evaluated 12 females and 3 males with an average age of 27 using digital video fluoroscopy [3]. Subjects wore a lead harness to improve quality of the image and stood at 10 degrees lumbar hyperextension before actively bending forward to full flexion and then returning to 10 degrees hyperextension [3]. The subjects were asked to complete this motion within 10 to 15 seconds in order to prevent radiation overexposure and to satisfy the sampling rate of the fluoroscope [3]. Dvorak et al. included 18 women with a mean age of 36 years and 23 men with a mean age of 39 years and used lateral roentgenographic examination [4]. Subjects stood in an upright position with their sacrum fixed to prevent hip motion and were asked to bend forward to maximum flexion, and then an examiner applied further flexion until the subject reported discomfort [4]. The same procedure was repeated for extension, and a lateral radiograph was taken at each end position [4]. Hayes et al. also used lateral roentgenographic examination on 59 individuals ranging from 19 to 59 years in age, but only active bending was evaluated while sitting with hips flexed 90 degrees during flexion and with the sacrum leaned against an edge of a table or chair during extension [5]. Pearcy et al. included only 11 males with an average age of 29.5 and used biplanar radiography [7]. Subjects were positioned in a rig that limited the motion of the pelvis and hips; lateral radiographs were taken while standing upright, at maximum flexion, and at maximum extension [7]. Ahmadi, Dvorak, and Pearcy reported the most variation at the L5-S level 
Table 5-1. Comparison of various methods of measuring intersegmental rotations in degrees.

\begin{tabular}{ccccccc}
\hline Author & Method & L1-L2 & L2-L3 & L3-L4 & L4-L5 & L5-S1 \\
\hline Panjabi and White 1978 & In vivo based on literature & 12.00 & 14.00 & 15.00 & 16.00 & 17.00 \\
Ahmadi 2009 & In vivo video fluoroscopy & 7.00 & 9.00 & 13.00 & 17.00 & 13.00 \\
Hayes 1989 & In vivo lateral radiographs & 7.00 & 9.00 & 10.00 & 13.00 & 14.00 \\
Pearcy 1984 & In vivo lateral radiographs & 13.00 & 14.00 & 13.00 & 16.00 & 14.00 \\
Dvorak 1989 & In vivo lateral radiographs & 11.90 & 14.50 & 15.30 & 18.20 & 17.00 \\
Panjabi 1994 & In vitro PM & 8.00 & 9.70 & 8.10 & 11.30 & 12.50 \\
Yamamoto 1989 & In vitro PM & 8.60 & 10.00 & 9.50 & 14.20 & 16.60 \\
Present study - PM & In vitro PM & 7.14 & 6.61 & 6.85 & 10.59 & 9.57 \\
Renner 2006 & In vitro PM + FL & 6.50 & 7.00 & 8.00 & 10.00 & 9.00 \\
Wong 2009 & In vitro EL & 7.90 & 6.10 & 8.00 & 7.60 & 8.90 \\
Present study - EL & In vitro EL & 5.65 & 5.83 & 4.62 & 5.73 & 6.37 \\
Present study - CLM & In vitro CLM & 6.03 & 6.83 & 7.68 & 9.45 & 6.68 \\
\hline
\end{tabular}

Sources: White, A.A. and M.M. Panjabi, Clinical Biomechanics of the Spine. 2nd ed. 1990, Philadelphia: Lippincott. p. 86-113. Ahmadi, A., et al., Kinematic analysis of dynamic lumbar motion in patients with lumbar segmental instability using digital videofluoroscopy. European Spine Journal, 2009. 18(11): p. 1677-85. Hayes, M.A., et al., Roentgenographic evaluation of lumbar spine flexion-extension in asymptomatic individuals. Spine, 1989. 14(3): p. 327-31. Pearcy, M., I. Portek, and J. Shepherd, Threedimensional $x$-ray analysis of normal movement in the lumbar spine. Spine, 1984. 9(3): p. 294-7. Dvorak, J., et al., Functional radiographic diagnosis of the lumbar spine. Flexion-extension and lateral bending. Spine, 1991. 16(5): p. 562-71. Panjabi, M.M., et al., Mechanical behavior of the human lumbar and lumbosacral spine as shown by three-dimensional load-displacement curves. The Journal of Bone and Joint Surgery, 1994. 76(3): p. 413-24. Yamamoto, I., et al., Three-dimensional movements of the whole lumbar spine and lumbosacral joint. Spine, 1989. 14(11): p. 1256-60. Renner, S.M., et al., Novel model to analyze the effect of a large compressive follower pre-load on range of motions in a lumbar spine. Journal of Biomechanics, 2007. 40(6): p. 1326-32. Wong, P., Biomechanical Comparison of Lumbar Disc Replacement. Master's Thesis. 2009, University of Tennessee Health Science Center. [1$5,7,9,10,30]$. 
most likely due to radiograph quality due to pelvis obstruction and to varying lordosis of subjects $[3,4,7]$. Panjabi and White have reported a composite motion profile that they believe to be the most representative values based on careful review of the literature [1].

Wong's eccentric loading study included seven whole lumbar specimens with an average age of 43, and specimens were mounted with the superior end plate of L1 horizontal [30]. Wong applied flexion and extension moments of 7.5 Nm using a lever arm similar to methods of the present study eccentric loading protocol [30]. The differences seen between the two eccentric loading studies could be due to differences in specimens since both studies had relatively small sample sizes. Panjabi's study included nine whole lumbar spines with an average age of 51, but four of these spines did not include the L1 level [2]. Yamamoto's study included ten whole lumbar specimens with an age range of 25 to 63, but the ages of three specimens were unknown [10]. Both Panjabi and Yamamoto mounted the specimens with the superior end plate of L4 horizontal and used cable driven pulleys to apply a maximum moment of $10 \mathrm{Nm}$, but rotations recorded at $7.5 \mathrm{Nm}$ were used in this comparison $[2,10]$. The studies of Yamamoto and Panjabi employed similar methods except that Panjabi applied a $100 \mathrm{~N}$ preload along the longitudinal axis of the specimen [2]. Not surprisingly, the two motion profiles show very similar trends with a decrease in rotation at the L3-L4 level which could be explained by the horizontal L4 mounting method. The validity of cable driven pulley methods of applying pure moments has recently been challenged after a study showed that fixed pulleys cause non-uniform loading conditions and produce non-trivial shear loads which deviates from the standard requirements of pure moment [17]. The pure moment methods of the present study overcomes the limitations of the previous pulley methods by minimizing shear and axial loads while applying a constant moment using horizontal, vertical, and rotational actuators of the spine robot. The shear and axial loads were confirmed to be minimal using load cells placed above and below the specimen, whereas Yamamoto and Panjabi do not describe the placement of a load cell to confirm the consistent application of pure moment $[2,10]$. Without the limitations of previous pure moment methods, the present study PM protocol is more accurate and also compares favorably with in vivo motion profiles.

The follower load study by Renner et al. included ten lumbar spines with an average age of 58 years and applied a flexion moment of $8 \mathrm{Nm}$ and an extension moment of $6 \mathrm{Nm}$ with a follower load of $800 \mathrm{~N}$ to represent the average load while standing [9]. The $800 \mathrm{~N}$ follower load path was placed along the center of rotation of each vertebra while mounted in the neutral upright position [9]. However, this placement may not be accurate for both modes of bending since it has been shown that the center of rotation of each motion segment shifts anteriorly during flexion and posteriorly during extension [21]. The misplacement of the follower load path during testing could actually be creating artificial moments at each level which would deviate from the uniformity of pure moment. The amount of $800 \mathrm{~N}$ to represent average load is also questioned because Rohlmann's in vitro study found that follower loads in the range of $280 \mathrm{~N}$ is enough to stabilize the spine [20]. This $280 \mathrm{~N}$ corresponds to the partial body weight above L1 of the average person which is comparable to the $300 \mathrm{~N}$ vertical load applied in the present study which showed no signs of buckling during compression. Since individual rotations 
maintained the same polarity during the CLM protocol, follower load may not be necessary when applying high loads in vitro. Despite differences in loading, the present study CLM motion profile was similar to the trend of Renner. The effect of applying more moment during flexion was not shown since Renner only reported range of motion as flexion plus extension, but the reduction of moment in extension could have affected the range of motion comparison. 


\section{CHAPTER 6. LIMITATIONS, CONCLUSIONS, AND FUTURE WORK}

The present study was limited to only flexion and extension modes of bending. Although rotations were limited to the sagittal plane, all three degrees of freedom were explored in terms of loading conditions. Pure moment involved one degree with the application of only a rotational load with minimal axial and shear loads. The pure moment protocol used with the Spine Robot overcame the limitations of standard fixed pulley methods of pure moment. Eccentric loading involved two degrees with the application of moment and axial loads. One limitation of the EL protocol is that the Spine Robot was not used, but testing still remained in the same group of specimens. Also, shear loads were not verified during EL but were assumed to be negligible due to the frictionless lever arm. Combined loading moment involved all three degrees of freedom with moment, axial, and shear loads via the application of a $300 \mathrm{~N}$ vertical load.

Each protocol included at least one load component different from the other two protocols which allowed the effects of each component to be distinguished based on the different range of motions produced by each protocol. Axial load was determined to limit range of motion. Shear loads appeared to increase flexion range of motion, but appeared to only increase extension range of motion until the facets joints prevented further motion. There is little information on the application and magnitude of lumbar shear loads in the literature, and further exploration of the effects of shear load on range of motion is necessary since the in vivo mechanics of the lumbar spine include shear. Specifically, the effects of sagittal lordosis, disc height, and disc angle on shear load should be explored.

The results of CLM show that follower load is not necessary to apply loads of in vivo magnitudes. CLM applies more realistic loading condition since follower load methods do not incorporate shear loads. However, one limitation of the CLM protocol is that it does not take into account muscle activity. CLM was also limited in the present study to only $300 \mathrm{~N}$ whereas follower load methods have applied up to $1200 \mathrm{~N}$. Future work with CLM should attempt to increase the magnitude of the vertical load.

Additionally, future work with CLM should include lateral, axial, and coupled rotations.

In vivo angular measurements may not be as accurate as in vitro measurements, and a comparison to both is necessary for validation. Comparison to previous multi-body lumbar data sets is limited to combined flexion and extension motion profiles due to a lack of data sets that separate flexion from extension. The motion profiles of the three methods in this study compared favorably to motion profiles of similar in vitro methods. Any discrepancies seen were attributed to differences in specimen preparation and mounting, end limit of motion, and testing methods. The variation seen among in vivo data sets was attributed to sample size, end limit of motion, passive versus active motion, initial subject positioning, and radiographic image quality. Newer methods of measuring range of motion using MRI or other more accurate methods may provide better in vivo data sets. 
Comparison to previous in vivo and in vitro data sets supports the use of the Spine Robot for multi-body lumbar spine studies. The Spine Robot is also able to apply pure moment methods without the limitations of fixed pulley pure moment methods. This study also introduced a new method of CLM that can be used to replicate more physiologic loading conditions. The multi-axis robot can be used as a universal platform to compare various methodologies by focusing on ROM. 


\section{LIST OF REFERENCES}

1. White, A.A. and M.M. Panjabi, Clinical Biomechanics of the Spine. 2nd ed. 1990, Philadelphia: Lippincott. p. 86-113.

2. Panjabi, M.M., et al., Mechanical behavior of the human lumbar and lumbosacral spine as shown by three-dimensional load-displacement curves. The Journal of Bone and Joint Surgery, 1994. 76(3): p. 413-24.

3. Ahmadi, A., et al., Kinematic analysis of dynamic lumbar motion in patients with lumbar segmental instability using digital videofluoroscopy. European Spine Journal, 2009. 18(11): p. 1677-85.

4. Dvorak, J., et al., Functional radiographic diagnosis of the lumbar spine. Flexionextension and lateral bending. Spine, 1991. 16(5): p. 562-71.

5. Hayes, M.A., et al., Roentgenographic evaluation of lumbar spine flexionextension in asymptomatic individuals. Spine, 1989. 14(3): p. 327-31.

6. Lee, S., et al., Development and validation of a new technique for assessing lumbar spine motion. Spine, 2002. 27(8): p. E215-20.

7. Pearcy, M., I. Portek, and J. Shepherd, Three-dimensional x-ray analysis of normal movement in the lumbar spine. Spine, 1984. 9(3): p. 294-7.

8. Patwardhan, A.G., et al., A follower load increases the load-carrying capacity of the lumbar spine in compression. Spine, 1999. 24(10): p. 1003-9.

9. Renner, S.M., et al., Novel model to analyze the effect of a large compressive follower pre-load on range of motions in a lumbar spine. Journal of Biomechanics, 2007. 40(6): p. 1326-32.

10. Yamamoto, I., et al., Three-dimensional movements of the whole lumbar spine and lumbosacral joint. Spine, 1989. 14(11): p. 1256-60.

11. Keorochana, G., et al., Effect of sagittal alignment on kinematic changes and degree of disc degeneration in the lumbar spine an analysis using positional MRI. Spine, 2011. 36(11): p. 893-8.

12. Adams, M.A., The Biomechanics of Back Pain. 2003, Edinburgh; New York: Churchill Livingstone. p. 19.

13. Nordin, M. and V.H. Frankel, Basic Biomechanics of the Musculoskeletal System. 3rd ed. 2001, Philadelphia: Lippincott Williams \& Wilkins. p. 268. 
14. Zufelt, N., A Kinematics-Based Testing Protocol to Study the Mechanics of the Human Lumbar Spine. Master's Thesis. 2008, The University of Tennessee Health Science Center.

15. Ochia, R.S., et al., Three-dimensional in vivo measurement of lumbar spine segmental motion. Spine, 2006. 31(18): p. 2073-8.

16. Bible, J.E., et al., Normal functional range of motion of the lumbar spine during 15 activities of daily living. Journal of Spinal Disorders \& Techniques, 2010. 23(2): p. 106-12.

17. Eguizabal, J., et al., Pure moment testing for spinal biomechanics applications: Fixed versus sliding ring cable-driven test designs. Journal of Biomechanics, 2010. 43(7): p. 1422-5.

18. Wilke, H.J., et al., Is it possible to simulate physiologic loading conditions by applying pure moments? A comparison of in vivo and in vitro load components in an internal fixator. Spine, 2001. 26(6): p. 636-42.

19. Patwardhan, A.G., et al., Effect of compressive follower preload on the flexionextension response of the human lumbar spine. Journal of Orthopaedic Research, 2003. 21(3): p. 540-6.

20. Rohlmann, A., et al., Influence of a follower load on intradiscal pressure and intersegmental rotation of the lumbar spine. Spine, 2001. 26(24): p. E557-61.

21. Fraysur, K.D., A Passive Pure Moment Protocol for Testing Spine Segments: Development and Application. Master's Thesis. 2010, University of Tennessee Health Science Center.

22. Chen, J., Development of a Flexible Biomechanical Testing Apparatus. Master's Thesis. 1996, University of Tennessee Health Science Center.

23. DiAngelo, D.J. and K.T. Foley, An improved biomechanical testing protocol for evaluating spinal arthroplasty and motion preservation devices in a multilevel human cadaveric cervical model. Neurosurgical Focus, 2004. 17(3): p. 22-9.

24. Kelly, B.P., et al., Biomechanical testing of a novel four-rod technique for lumbopelvic reconstruction. Spine, 2008. 33(13): p. E400-6.

25. Goel, V.K., et al., Biomechanical testing of the spine. Load-controlled versus displacement-controlled analysis. Spine, 1995. 20(21): p. 2354-7.

26. Panjabi, M.M., Biomechanical evaluation of spinal fixation devices: I. A conceptual framework. Spine, 1988. 13(10): p. 1129-34. 
27. Panjabi, M.M., Hybrid multidirectional test method to evaluate spinal adjacentlevel effects. Clinical Biomechanics, 2007. 22(3): p. 257-65.

28. Kelly, B., A Multiaxis Programmable Spine Robot for the Study of Multibody Spinal Biomechanics Using Real-Time Hybrid Force and Displacement Control Strategies. Doctoral Dissertation. 2005, The University of Tennessee Health Science Center.

29. Cholewicki, J. and S.M. McGill, Mechanical stability of the in vivo lumbar spine: Implications for injury and chronic low back pain. Clinical Biomechanics, 1996. 11(1): p. 1-15.

30. Wong, P., Biomechanical Comparison of Lumbar Disc Replacement. Master's Thesis. 2009, University of Tennessee Health Science Center. 
APPENDIX A. RADIOGRAPHS
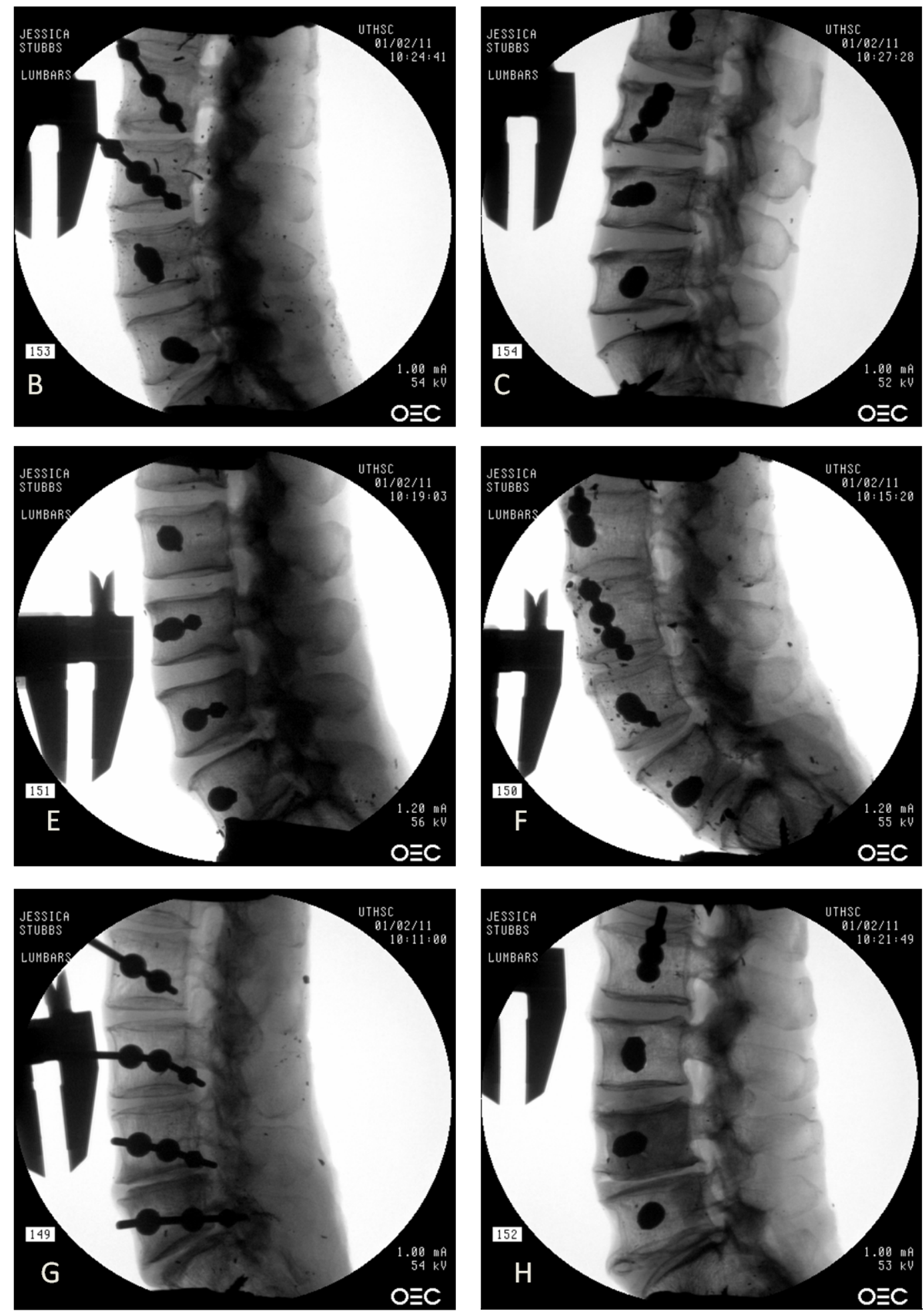

Figure A-1. Radiographs of specimens B, C, E, F, G, and $\mathrm{H}$ after rod placement. 


\section{APPENDIX B. FLEXIBILITY CURVES}
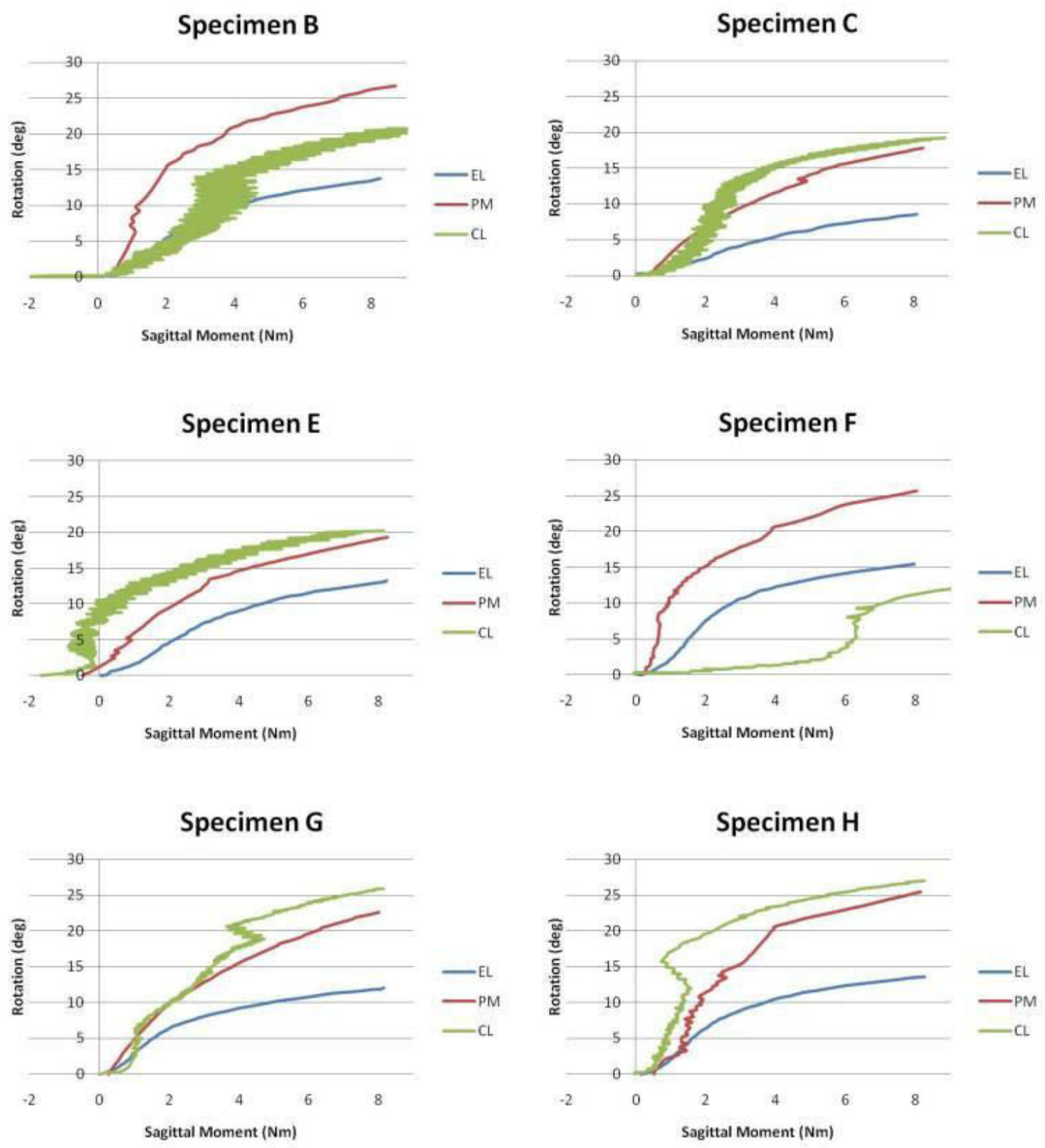

Figure B-1. Flexibility curves for each specimen during flexion. 

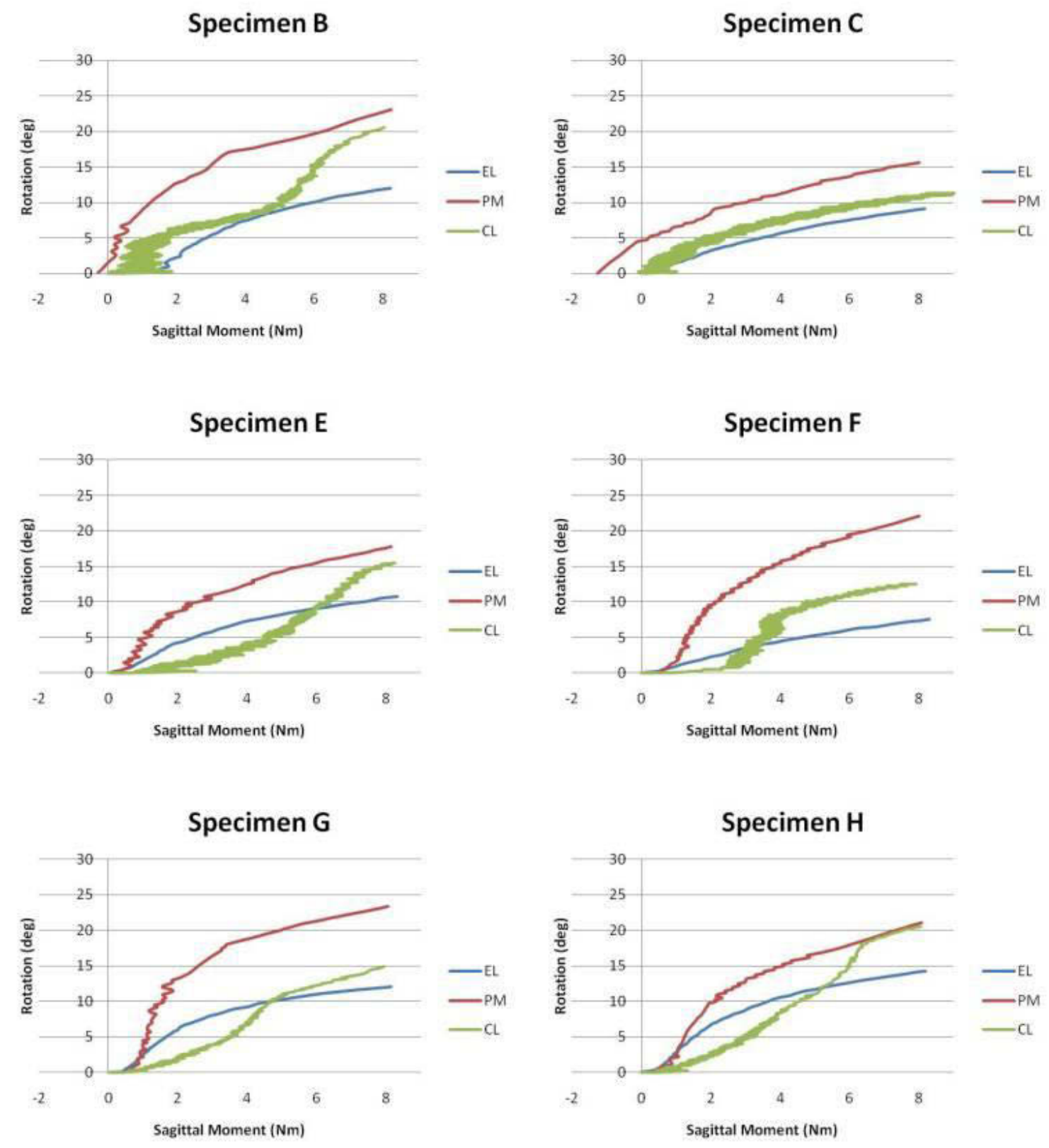

Figure B-2. Flexibility curves for each specimen during extension. 


\section{VITA}

Jessica Rose Stubbs was born in Memphis, Tennessee in 1986. She was raised in Germantown, Tennessee where she attended Houston High School. Upon graduation in 2004, she decided to follow both of her parents' footsteps by attending Mississippi State University in Starkville, Mississippi. She graduated summa cum laude with a Bachelor of Science in Biological Engineering. After graduation in May 2008, she worked as an intern in manufacturing engineering at Smith and Nephew in Memphis, Tennessee while also enrolled as graduate student at the University of Tennessee Health Science Center and University of Memphis Joint Program in Biomedical Engineering. Jessica entered the program as a student of Dr. Waleed Gaber to study the biomechanics of wound healing. In January of 2009, she joined Dr. Denis DiAngelo's Joint Implant Biomechanics Lab to continue studying biomechanics in the UTHSC program. In May 2011, Jessica earned a Master of Science in Biomedical Engineering focusing on the biomechanics of the lumbar spine. 